\title{
Expression of tachykinin receptors (tacr1a and tacr1b) in zebrafish: influence of cocaine and opioid receptors
}

\section{Roger López-Bellido, Katherine Barreto-Valer and Raquel E Rodríguez}

Department of Biochemistry and Molecular Biology, Institute of Neuroscience of Castilla y León, University of Salamanca, C/Pintor Fernando Gallego No. 1, Lab-13, 37007 Salamanca, Spain
Correspondence should be addressed to R E Rodríguez

Email

requelmi@usal.es

\begin{abstract}
Opioid and tachykinin receptors (TACRs) are closely related in addiction and pain processes. In zebrafish, opioid receptors have been cloned and characterized both biochemically and pharmacologically. However, the tacr1 gene has not yet been described in zebrafish. The aim of this research was to identify the tacr1 gene, study the effects of cocaine on tacr1, and analyze the interaction between tacr 1 and opioid receptors. We have identified a duplicate of tacr1 gene in zebrafish, designated as tacr1a and tacr1b. Phylogenetic analyses revealed an alignment of these receptors in the Tacr1 fish cluster, with a clear distinction from other TACR 1s of amphibians, birds, and mammals. Our qPCR results showed that tacr1a and tacr $1 b$ mRNAs are expressed during embryonic development. Whole-mount in situ hybridization showed tacr 1 expression in the CNS and in the peripheral tissues. Cocaine $(1.5 \mu \mathrm{M})$ induced an upregulation of tacr $1 \mathrm{a}$ and $\operatorname{tacr} 1 \mathrm{~b}$ at 24 and $48 \mathrm{~h}$ post-fertilization (hpf; except for tacr $1 \mathrm{a}$ at $48 \mathrm{hpf}$, which was downregulated). By contrast, HEK-293 cells transfected with tacr1a and tacr $1 \mathrm{~b}$ and exposed to cocaine showed a downregulation of tacr1s. The knockdown of ZfDOR2 and ZfMOR, opioid receptors, induced a down- and upregulation of tacr1a and $\operatorname{tacr} 1 b$ respectively. In conclusion, tacr $1 a$ and $\operatorname{tacr} 1 b$ in zebrafish are widely expressed throughout the CNS and peripherally, suggesting a critical role of these tacr1s during embryogenesis. tacr1a and tacr $1 b$ mRNA expression is altered by cocaine exposure and by the knockdown of opioid receptors. Thus, zebrafish can provide clues for a better understanding of the relationship between tachykinin and opioid receptors in pain and addiction during embryonic development.
\end{abstract}

\section{Key Words}

- TACR1 gene

- opioid receptors

- cocaine

- zebrafish

\section{Introduction}

The biological actions of the tachykinin family - substance $\mathrm{P}$ (SP), neurokinin A (NKA) and NKB, hemokinin-1, and endokinins - are mediated by transmembrane G-proteincoupled receptors (GPCRs) and they have been classified within the $\mathrm{NK}_{1}, \mathrm{NK}_{2}$, and $\mathrm{NK}_{3}$ receptor types (Regoli et al. 1994, Maggi 2000, Harrison \& Geppetti 2001, Pennefather
Journal of Molecular

Endocrinology

(2013) 50, 115-129 et al. 2004), which are encoded by tachykinin receptor 1 (TACR1), TACR2, and TACR3 genes respectively (Zhou et al. 2012). SP, $\mathrm{NKA}$, and $\mathrm{NKB}$ bind to $\mathrm{NK}_{1}, \mathrm{NK}_{2}$, and $\mathrm{NK}_{3}$ receptors (also named TACR1, TACR2, and TACR3) respectively (Regoli et al. 1987, Regoli et al. 1994, DeVane 2001, Harrison \& Geppetti 2001). $\mathrm{NK}_{1}$ receptor $\left(\mathrm{NK}_{1} \mathrm{R}\right)$ is 
considered the SP receptor (Maggi 1995). Several studies have shown that $\mathrm{NK}_{1}$ is widely distributed throughout the CNS (basal ganglia, dorsal tegmental areas, inferior colliculus, olfactory bulb, hypothalamus, hippocampus, substantia nigra, cerebral cortex, septum, striatum, mesencephalon, medulla oblongata, and the dorsal horn of the spinal cord; Shults et al. 1984, Maeno et al. 1993) and its activation has been implicated in different processes such as pain, synaptic transmission, neurogenic inflammation, neurotoxicity, mood disorder, stress, anxiety, and addiction (Regoli et al. 1994, Harrison \& Geppetti 2001, Yu et al. 2002, Gadd et al. 2003, Gamboa-Esteves et al. 2004, Commons 2010).

The analgesic effect of opioids is mediated mainly by MOR (Matthes et al. 1996) but, as several studies have shown that MOR and $\mathrm{NK}_{1} \mathrm{R}$ are co-localized (Aicher et al. $2000 a, b)$ and present interaction between them (Pfeiffer et al. 2003), it is possible that analgesic effects of MOR can be modified by $\mathrm{NK}_{1} \mathrm{R}$ or vice versa. Moreover, in vitro experiments reported that MOR and $\mathrm{NK}_{1} \mathrm{R}$ form heterodimers in HEK-293 $\left(\mathrm{NK}_{1}-\mathrm{MOR}\right)$ where the activation of either type, by SP and DAMGO respectively, induces endocytosis (Pfeiffer et al. 2003). Besides, it has also been described that $\mathrm{NK}_{1} \mathrm{R}$ activation by SP inhibits the endocytosis of MOR ( $\mathrm{Yu}$ et al. 2009), indicating that SP and its receptor are involved in the modulation of MOR. Several studies have also shown that tachykinin and opioid systems are related in the addiction process, as $\mathrm{NK}_{1} \mathrm{R}$ and MOR are localized in different reward-related regions (Nakaya et al. 1994, Pickel et al. 2000, Garzón \& Pickel 2001, Gadd et al. 2003, Laurent et al. 2012). Likewise, it has been suggested that SP acts in a similar manner to cocaine, producing an increase in dopamine neurotransmitter levels in the synaptic cleft (Kombian et al. 2009).

The tachykinin system has been cloned and characterized both biochemically and pharmacologically in different species (for reviews, see Maggi (1995) and Pennefather et al. (2004)), and numerous investigations using the zebrafish have proven that this organism is a valuable vertebrate animal model for study of human diseases, pharmacology and drug discovery, and development studies in vertebrates (Chakraborty et al. 2009, Lohi et al. 2012, Santoriello \& Zon 2012). The study of the tachykinin system using the zebrafish could improve our understanding of different clinical situations where the tachykinin system is involved. This will also help to better understand of the interaction of the tachykinin system with other systems, like the opioids system that are involved in pain and addiction.
To date, the tachykinin system has not been described completely in zebrafish. Recently, the tac1, tac2, and tac 3 precursors and the Tacr3s (Tacr3a1, Tacr3a2, and Tacr3b; Ogawa et al. 2012, Zhou et al. 2012) have been cloned. Taking the above into consideration, we aimed to i) clone the tacr 1 gene and study its expression during embryogenesis, ii) determine the evolutionary history of the tacr1s using phylogenetic analyses, iii) investigate the effects of cocaine on the tacr1s, and iv) study the interrelationship between the tachykinin system and opioid receptors during embryonic development.

\section{Materials and methods}

\section{Animals}

Adult zebrafish (AB strain) were maintained on a $14 \mathrm{~h}$ light: $10 \mathrm{~h}$ darkness cycle at $26^{\circ} \mathrm{C}$ at our Fish Facilities. Embryos were raised at $28.5^{\circ} \mathrm{C}$ and maintained in dishes in $\mathrm{E} 3$ medium $(5 \mathrm{mM} \mathrm{NaCl}, 0.17 \mathrm{mM} \mathrm{KCl}, 0.33 \mathrm{mM} \mathrm{CaCl}$, and $0.33 \mathrm{mM} \mathrm{MgSO}_{4}$ in distilled water; Sigma). Embryo ages were expressed as hours post-fertilization (hpf). All procedures and experimental protocols were carried out in accordance with the guidelines of the European Communities Council directive of 24 November 1986 (86/609/EEC), and to the current Spanish legislation for the use and care of animals (BOE 252/34367-91, 2005).

\section{Drug treatment}

Zebrafish embryos at $5 \mathrm{hpf}$ were exposed to $1.5 \mu \mathrm{M}$ cocaine hydrochloride $(\mathrm{HCl})$ and were then collected at 24 and $48 \mathrm{hpf}$; both stages are important during zebrafish embryonic development: at $24 \mathrm{hpf}$, the CNS is being formed and differentiated and primary organogenesis is finished at $24 \mathrm{hpf}$. HEK-293 cells (expressing zebrafish TACRs) were treated with $1.5 \mu \mathrm{M}$ cocaine $\mathrm{HCl}$ over $24 \mathrm{hpf}$. The doses of $1.5 \mu \mathrm{M}$ cocaine $\mathrm{HCl}$ was chosen as this dose is comparable to the concentration present in human neonates (Dempsey et al. 1999).

\section{Cloning of tacr1a and tacr1b receptors in zebrafish}

The PCR program used for tacr $1 a$ and tacr $1 b$ amplification was $5 \mathrm{~min}$ at $95^{\circ} \mathrm{C}$, followed by 35 cycles of $1 \mathrm{~min}$ at $95^{\circ} \mathrm{C}$, $1 \mathrm{~min}$ at $59^{\circ} \mathrm{C}$ (for tacr $1 a$ ) and $62^{\circ} \mathrm{C}$ (for tacr $1 b$ ), and $3 \mathrm{~min}$ at $72{ }^{\circ} \mathrm{C}$ and a final extension temperature of $72{ }^{\circ} \mathrm{C}$ for $10 \mathrm{~min}$. PCR primers (Table 1) were based on the predicted sequences of the tacr1a and tacr1b genes from PubMed (XM_687377.2, XM_001343037.3). The desired tacr1 $a$ and

Published by Bioscientifica Ltd. 
Table 1 Oligonucleotides used in this study

\begin{tabular}{l} 
Genes \\
\hline ef1a \\
tacr1a (qPCR) \\
tacr1b (qPCR) \\
tacr1a (riboprobe) \\
tacr1b (riboprobe) \\
tacr1a-pEGFP-C3 \\
tacr1b-pDsRed1-N1 \\
GAPDH
\end{tabular}

\begin{tabular}{l} 
Forward \\
\hline GTACTTCTCAGGCTGACTGTG \\
CGCTATTGCGCTCGACAGA \\
CCTGCTGGCTCCCCTATCA \\
CCTCCAGTCAAGAAACAATCGCT \\
TCCCCTTGTTAAAATGCCTGTTCT \\
${ }^{\text {a }}$ ctgtaCTCGAGGATTCGTTCATCACTTCC ${ }^{\text {b }}$ \\
atgataAAGCTTATGGATCCGCTGTACATCAC \\
ATGAGAAGTATGACAACAGCCT
\end{tabular}

\begin{tabular}{l} 
Reverse \\
\hline ACGATCAGCTGTTTCACTCC \\
GCTACATTGACTGGCCCGA \\
GGAATCCCGCTCGAAACCT \\
GGTTGGCATTCGTTTTCCATGAC \\
ACCGTTTTGGAGCTCACTAGC \\
a tcgtaGGTACCTCATTCCTGTAGGTTATTAC \\
a agataCCGCGGTGCTACGTTGTTACTGGAAT \\
CAGTGATGGCATGGACTGTG
\end{tabular}

\begin{tabular}{|c|c|}
\hline Amplicon & $\boldsymbol{T}\left({ }^{\circ} \mathrm{C}\right)$ \\
\hline 136 & 55 \\
\hline 186 & 55 \\
\hline 173 & 55 \\
\hline 1364 & 59 \\
\hline 1506 & 62 \\
\hline 1278 & 64 \\
\hline 1236 & 64 \\
\hline 138 & 57 \\
\hline
\end{tabular}

a Lower-case letters represent adaptador sequences.

${ }^{b}$ The nucleotides underlined are recognition sites of the Xhol and Kpnl restriction enzymes respectively.

'Nucleotide sequences underlined are specific sites of recognition for HindIII and Sacll restriction enzymes respectively.

tacr1b amplicons were subcloned using the pCRII vector (Invitrogen). TOP $10^{\prime} \mathrm{F}$ cells (Invitrogen) were transformed with the constructs, and miniprep (ZYMO Research Corporation, CA, USA) and midiprep (Sigma) were performed. These constructs of both tacr1a and tacr $1 b$ with pCRII were digested with KpnI and EcoRV for $1 \mathrm{~h}$ at $37^{\circ} \mathrm{C}$. Then, the purified products were sequenced using the T7 and SP6 sequencing primers.

\section{Phylogenetic analyses}

The prediction of the open reading frames (ORFs) and translations of tacr $1 a$ and tacr $1 b$ into amino acid sequences were performed using the ORF Finder program from SMS (http://www.bioinformatics.org/sms2/). The Tacr1a and Tacr1b amino acid sequences from zebrafish and other orthologs of several vertebrates were aligned in NBRF format using the ClustalW Software. Molecular Evolutionary Genetic Analysis (MEGA 5) was used to construct neighbor-joining (NJ) and maximum likelihood (ML) trees.

\section{Expression of tacr1a and tacr1b from zebrafish in mammalian HEK-293 cells}

In order to insert the DNA sequences of both tacr1a and tacr $1 b$ in-frame with the vector sequences, two restriction endonuclease (RE) sites from the multiple cloning sites of the plasmid vector were chosen: XhoI and KpnI for pEGFPC3 (Clontech) and HindIII and SacII for pDsRed1-N1 (Clontech). The RE recognition sequences were added to the ends of the primers (extreme $5^{\prime}$-terminal), which were used to amplify the tacr $1 a$ and tacr $1 b$ (Table 1). The vector and inserted genes were digested with both REs in a double digest for $1 \mathrm{~h}$ at $37^{\circ} \mathrm{C}$, and after their respective purification, the inserts were ligated using the TA cloning Kit (Invitrogen) in the plasmid vectors pEGFP-C3 (GFP) and pDs1Red-N1 (DsRed). The PCR program used for tacr $1 a$ and tacr $1 b$ amplification was $5 \mathrm{~min}$ at $95^{\circ} \mathrm{C}$, followed by 35 cycles of $45 \mathrm{~s}$ at $95{ }^{\circ} \mathrm{C}, 45 \mathrm{~s}$ at $64^{\circ} \mathrm{C}$, and $3 \mathrm{~min}$ at $72{ }^{\circ} \mathrm{C}$ and a final extension temperature of $72{ }^{\circ} \mathrm{C}$ for $10 \mathrm{~min}$. Plasmid transfection was accomplished using the Effectene Transfection Reagent Kit (Qiagen). The expression vectors encoded neomycin/kanamycin resistance, which permitted us to select and obtain stable clones of tacr $1 a$ and tacr $1 b$ with $0.5 \mathrm{mg} / \mathrm{ml}$ geneticin (Gibco BRL). After an 2-month selection period, stably transfected HEK-293 cells expressing tacr1a-GFP and tacr1b-DsRed were grown in DMEM supplemented with $10 \%$ FCS, $100 \mathrm{U} / \mathrm{ml}$ penicillin, $0.1 \mathrm{mg} / \mathrm{ml}$ streptomycin, and $0.25 \mathrm{mg} / \mathrm{ml}$ geneticin at $37^{\circ} \mathrm{C}$ under a $5 \% \mathrm{CO}_{2}$ atmosphere. HEK-293 cells expressing tacr1a-GFP and tacr $1 b$-DsRed were exposed to $1.5 \mu \mathrm{M}$ cocaine $\mathrm{HCl}$ for $24 \mathrm{hpf}$.

\section{RNA extraction, retrotranscription, and real-time PCR (qPCR)}

Total RNA from the embryos and HEK-293 cells was extracted using TRIzol reagent (Invitrogen), following the protocol recommended by the manufacturers. In all cases, RNA samples were treated with DNase I (Roche) at $37^{\circ} \mathrm{C}$ for $15 \mathrm{~min}$. The quantity and quality of the RNA were determined by measuring absorbance at $260 \mathrm{~nm}$ and $260 \mathrm{~nm} / 280 \mathrm{~nm}$, using NanoDrop 2000C (Thermo Fisher Scientific, Waltham, MA, USA). cDNA synthesis was carried out by retrotranscription (RT) of total RNA to cDNA using the Promega RT Kit, following the protocol recommended by the manufacturers. The cDNA samples were then treated with RNase A ( $20 \mathrm{ng} / \mu \mathrm{l}$ for $7-8 \mu \mathrm{g}$ cDNA) for $20 \mathrm{~min}$ at $37^{\circ} \mathrm{C}$. Following this, these products were purified using the QIAquick PCR Purification Kit (Qiagen) and eluted in DNase-free water. The oligonucleotides used for qPCR experiments are listed in Table 1. qPCR products

Published by Bioscientifica Ltd. 
were measured using the curve of the SYBR-Green method. SYBR-Green was included in a $2 \times$ Master Mix from Applied Biosystems (SYBR Green dye, dNTPs, Passive Reference (ROX), Amplitaq1 Gold DNA polymerase). The ABI Prism 7300 detection system (Applied Biosystems) was used to amplify the different genes. The final volume of each reaction was $20 \mu \mathrm{l}$ : $10 \mu \mathrm{l}$ Master Mix, $1 \mu \mathrm{l}$ of each oligonucleotide, $7 \mu \mathrm{l}$ distilled water, and $1 \mu \mathrm{l}$ cDNA at a concentration of $5 \mathrm{ng} / \mu \mathrm{l}$.

Standard curves for absolute quantification were performed for the different genes studied in zebrafish embryos. A basic PCR method was implemented to amplify the fragments of the different genes to be studied. PCR products were visualized on agarose gel and the fragment corresponding to each gene was cut from the agarose gel and purified. Serial 1:10 dilutions were made from the purified PCR product, ranging from $10^{-2}$ to $10^{-5} \mathrm{ng} / \mu \mathrm{l}$ and chosen according to their cycle quantification $(\mathrm{Cq})$ values and considering that perfect amplification had been obtained when the difference in the $\mathrm{Cq}$ values from one dilution to the next was close to 3.3 , which afforded a four-point straight line with a slope of -3.3 .

\section{Spatial expression of tacr1a and tacr1b mRNA}

The constructs of both tacr1a and tacr1b with pCRII were linearized by EcoRV and KpnI digestion respectively over $1 \mathrm{~h}$ at $37^{\circ} \mathrm{C}$. From the linearized tacr $1 a$ and tacr $1 b$ DNAs, antisense RNAs were transcribed with T7 and SP6 promoters respectively and the RNAs were also labeled with digoxigenin. These latter products were treated with DNase I (Roche) and purified. The riboprobe $(600 \mathrm{ng} / \mathrm{ml})$ was diluted in the prehybridization solution for the in situ hybridization (ISH) experiments. Embryos at 24 and 48 hpf were dechorionated, fixed with $4 \%$ paraformaldehyde (PFA) in PBS overnight at $4{ }^{\circ} \mathrm{C}$, washed twice in PBS, 5 min each, at room temperature, and finally maintained in absolute methanol at $-20^{\circ} \mathrm{C}$ until use. The following day, the embryos were rehydrated in consecutive dilutions of methanol/PBS $(75,50$, and 25\%), $5 \mathrm{~min}$ for each dilution. These were washed four times for $5 \mathrm{~min}$ each in 100\% PBS-Tween 20 (0.2\%; PBT). Proteinase K $(10 \mu \mathrm{g} / \mathrm{ml})$ was used to permeabilize the embryos at room temperature over $20 \mathrm{~min}$ for $24 \mathrm{hpf}$ embryos and $40 \mathrm{~min}$ for $48 \mathrm{hpf}$ embryos. Proteinase $\mathrm{K}$ digestion was stopped by incubating the embryos for $20 \mathrm{~min}$ in $4 \%$ PFA in $1 \times$ PBS. Four washes, $5 \mathrm{~min} /$ wash, in $1 \times \mathrm{PBT}$ were performed to remove residual PFA. After $2 \mathrm{~h}$ of prehybridization with tacr $1 a$ and tacr $1 b$ riboprobe, the embryos were left overnight at $64{ }^{\circ} \mathrm{C}$ to hybridize. Washes were performed every $20 \mathrm{~min}$ in each wash solution with prehybridization/Tris-buffered saline (TBS; 50\%/50\%) and TBS-Tween 20 (0.2\%; TBST) over $2 \mathrm{~h}$. Then, the embryos were blocked with blocking buffer (goat serum + TBST) for $2 \mathrm{~h}$ and were incubated overnight with antidigoxigenin antibody conjugated with alkaline phosphatase (1:3000, Roche) at $4{ }^{\circ} \mathrm{C}$. The following day, the embryos were washed with Xpho solution (1 M Tris$\mathrm{HCl}, \mathrm{pH}$ 9.5, $1 \mathrm{M} \mathrm{MgCl}_{2}, 4 \mathrm{M} \mathrm{NaCl}$, and 20\% Tween 20) for $10 \mathrm{~min}$ over $1 \mathrm{~h}$, and finally, the hybridization was developed with fresh NBT/BCIP mix (Roche).

\section{Knockdown of opioid receptors}

Antisense morpholino oligonucleotides (MOs) of opioid receptors were used to knock down opioid receptors (Gene Tools, Philomath, OR, USA) in order to determine the relationship between opioid and TACRs (tacr1a and tacr $1 b$ expression). Approximately $3 \mathrm{nl}$ of MOs were microinjected into the yolks of zebrafish embryos at the one- to four-cell stage with a micromanipulator-microinjector system from Eppendorf AG (Hamburg, Germany). The MO experimental groups (ZfMOR, ZfDOR1, ZfDOR2, and ZfDOR1-ZfDOR2) were compared with the MO control groups (microinjected with a standard MO control solution and embryos without microinjections). The following MO sequences were used: ZfMOR (AATGTTGCCAGTGTTTTCCATCATG), ZfDOR1 (GAATGACGGACGGCTCCATCGCTTC), and ZfDOR2 (GGAGGCTCCATTATGCTCGTCCCCT). The MO concentrations used for the different opioid receptors were $0.2 \mu \mathrm{M}$ (ZfMOR), $1 \mu \mathrm{M}$ (ZfDOR1), $1 \mu \mathrm{M}$ (ZfDOR2), and $1 \mu \mathrm{M}$ (ZfDOR1-ZfDOR2).

\section{Statistical analyses}

The results are presented as means of transcripts \pm s.E.M. of the values. Pairs of the data were analyzed by Student's $t$-test while multiple comparisons were evaluated by ANOVA. Follow-up analysis was performed using the Dunnet test for multiple comparisons. Differences were considered significant at $P<0.05$. All statistical analyses were performed with Prism 5 (GraphPad Software, Inc., La Jolla, CA, USA).

\section{Results}

\section{Full-length tacr1a and tacr1b cloning in zebrafish}

After RNA isolation from 48-hpf zebrafish embryos, PCR amplification, cloning, and sequencing, we obtained the full-length cDNA of two genes, which were designated

Published by Bioscientifica Ltd. 
taaaggatcactggagtaatcctgtattttaggggaacagaggcataccagttggaact cttctttgcgccatgaaactcaataatttgactttttactttaaagagaacatggtcgat tgaacattcataaaacctccagtcaagaaacaatcgctttactatttttccccaacagga 1 ATGGATTCGTTCATCACTTCCACTGACTTCCCAAGTAACTGGACAGTCAACTCCACCGCG

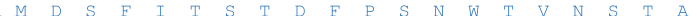

61 CAGAATGAAACCGAGATATACTGGAACCAGTTTGTTCAGCCGGTTTGGAGGATAGTGCTC $\begin{array}{llllllllllllllllllllll} & 1 & Q & N & E & T & E & I & Y & W & N & O & F & V & Q & P & V & W & R & I & V & L\end{array}$ 121 TGGGCAGTGGCTTACAGCACAATTGTCGTCGTCTCCGTGGTGGGCAATATTACGGTGATA

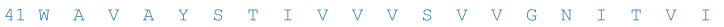
181 TGGATCATTTTGGCGCACAAACGCATGCGAACTGTGACGAATTATTTCCTAGTGAACTT

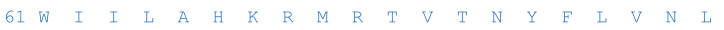
241 GCGTTCGCAGAAGCGTCAATGTCAGCGTTCAACACCGTAATAAACTTTGTCTATTCCGTA $\begin{array}{lllllllllllllllllllllllll}81 & A & F & A & E & A & S & M & S & A & F & N & T & V & I & N & F & V & Y & S & V\end{array}$ 301 CACAATGAGTGGTATTTCGGGCTGTATTACIGCAGAITCACAACTACITCCCCATTGCC 361 GCTGTCTTCGCCTCTATATATTCAATGACCGCTATTGCGCTCGACAGATATATGGCCATC $121 \mathrm{~A} \quad \mathrm{~V} \quad \mathrm{~F}$ A $\mathrm{S}$ I $\mathrm{Y}$ S 421 ATTCACCCCCTGCAGCAGAGGATGTCTGCGACCCAGACAAAGGTGGTGATCGGGGTCATC

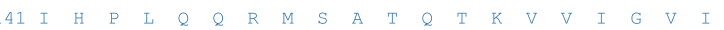
481 TGGATCCTGGCTCTTCTGCTGGCCTTCCCCCAATACTACTACTCCGACACTGACCAGCTC 161 W 541 CCCGGCAGGGTGGTCTGCTACATTGACTGGCCCGAGTACACTCTCCTGGACTTCAAAACA

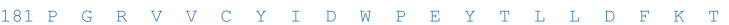
601 ATGTACTTTGTATGTGTAGCGGTGCTGATTTACTTCCTCCCTCTCCTGGTGATGGGCTGT

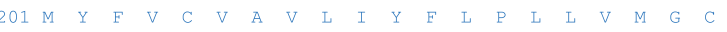
661 GCTTACCTGGTGGTGGGATTGACTCTGTGGGCCAGCGAGATCCCAGGAGACTCATCGGAC

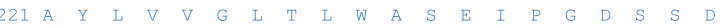
721 CGTTACCAAGAGCAGCTGACAGCCAAACGCAAGGTGGTGAAGATGATGATTGTGGTGGTG $\begin{array}{llllllllllllllllllll}241 & R & Y & Q & E & Q & L & T & A & K & R & K & V & V & K & M & M & I & V & V \\ 7\end{array}$ 781 TGTACGTTCGCCGTCTGCTGGCTTCCCTATCATGTCTATTTCCTGATCCACCAATTCTAC $\begin{array}{lllllllllllllllllllll}261 & C & \text { T } & \text { E } & \text { A } & \text { V } & C & \text { W } & \text { L } & \text { P } & \text { Y } & \text { H } & \text { V } & \text { Y } & \text { F } & \text { L } & \text { I } & \text { H } & Q & \text { F } & \text { Y }\end{array}$ CTATCATGTGGCTGGCA $\begin{array}{lllllllllllllllllllll}281 & P & H & L & F & E & H & T & F & I & Q & Q & V & Y & L & T & I & M & W & L & A\end{array}$ 901 ATGAGCTCCACCATGTACAACCCCATTATCTACTGCTGCCTCAATGACAGGTTTCGAGC

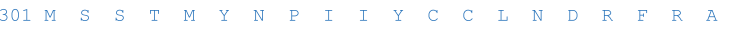
961 GGATTTAAACAAGCTTTCCGCTGCTGTCCCTGCGTCCCTGAAGGGTCCTACGAGGGTCTA $\begin{array}{lllllllllllllllllllll}321 & G & F & K & Q & A & F & R & C & C & P & C & V & P & E & G & S & Y & E & G & L\end{array}$ 1021 GAGCTCAAGTCCACTCGCTACCTTCAAACCCAGACAAGTCTGTACAGGGCCAGCCGCATG

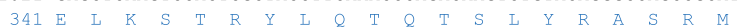
1081 GAAACCAGTGTGTCCTGCGTGATGCAGCCTAGTGAAGACGGCATAAGGTCACATTCGGG 361 E $\quad$ T $S$ S V S C C V 1141 AGCGTCAGAGGGGCTGCTGTGAAGCCAGCAGCTCGCAGCCCGTCCAGAGAGTTCACCTCC $\begin{array}{lllllllllllllllllllll}381 & \mathrm{~S} & \mathrm{~V} & \mathrm{R} & \mathrm{G} & \mathrm{A} & \mathrm{A} & \mathrm{V} & \mathrm{K} & \mathrm{P} & \mathrm{A} & \mathrm{A} & \mathrm{R} & \mathrm{S} & \mathrm{P} & \mathrm{S} & \mathrm{R} & \mathrm{E} & \mathrm{F} & \mathrm{T} & \mathrm{S}\end{array}$ 1201 AATGGCTCATCCTCTCGCAGCATCTCCAAAACCGTGTCGGAGACCTCCAGCTTCTACTCC

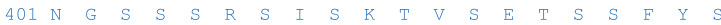
1261 GTAATAACCTACAGGAATGAgagcogagaaacacagtcatgraaaacgaatqccaacca $421 \mathrm{~S} N$ N L Q F Stop acgcttaattgattgatgtatcctctaacggcagtgtaaatgcgttttgaagctggtt gagtacacacacgctaagtgtttttaatttggagctatccatcatctttcattcttctc acattctattcattaggtttataattataattagaggaacatttcgattgattttgttt

C
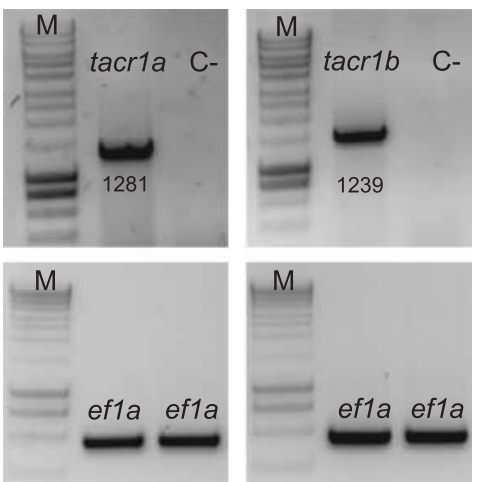

Figure 1

Nucleotide and deduced amino acid sequence of the $1281 \mathrm{bp}$ CDNAencoding zebrafish tacr1a receptor (A) and the 1239 bp cDNA-encoding zebrafish tacr $1 b$ receptor (B). The nucleotides are shown in black, amino acids in blue, and the $5^{\prime}$-UTR and $3^{\prime}$-UTR sequences in green. The forward

http://jme.endocrinology-journals.org DOI: 10.1530/JME-12-0199
(C) 2013 Society for Endocrinology Printed in Great Britain ccacgacaactgtgegcgegtttggagaggegcgctcgcgactcagcggatcetcagcgo atttcttcaactcatttttggaggctctataattcacaaaacaaacctcaaaacaacaaa acgccaacttccccttgttaaaatgcetgttctctgatatattagtccaccatggatceg 1 ATGGATCCGCTGTACATCACTTCGCCAAACTCGAGCGCCGATAACGGGAGCGCCGGTTCT $\begin{array}{lllllllllllllllllllll}1 & M & D & P & L & Y & I & T & S & P & N & S & S & A & D & N & G & S & A & G & S\end{array}$ 61 CCGGTAAACGACACGGAGGTGTTCGGGAACCAGTTCGTGCAGCCGGTCTGGCAGATCGTA

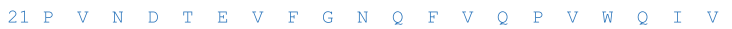
121 CTATGGGCTATCGCGTACTGCACTATAGTTCTGGTGTCGGTAGTGGGAAATATCACGGTG $\begin{array}{rllllllllllllllllllll}41 & \text { L } & \text { W } & \text { A } & \text { I } & \text { A } & \text { Y } & \text { C } & \text { T } & \text { I } & \text { V } & \text { L } & \text { V } & \text { S } & \text { V } & \text { V } & \text { G } & \text { N } & \text { I } & \text { T } & \text { V } \\ 181 & \text { ATCTGGATCATTTTAGCGGCATAAGCGCATGAGGACGGTCACCCAACTACTTTCTGGTGAAT }\end{array}$ 61 I W 241 CTGGCGTTCGCGGAGGCGTCCATGTCCGCCTTTAACACCGTCATTAACTTTATTTAACGCG $\begin{array}{lllllllllllllllllllll}81 & \mathrm{~L} & A & \mathrm{~F} & \mathrm{~A} & \mathrm{E} & \mathrm{A} & \mathrm{S} & \mathrm{M} & \mathrm{S} & \mathrm{A} & \mathrm{F} & \mathrm{N} & \mathrm{T} & \mathrm{V} & \mathrm{I} & \mathrm{N} & \mathrm{F} & \mathrm{I} & \mathrm{Y} & \mathrm{A}\end{array}$ 301 GTGCACAATGAGTGGTACTTCGGGCTGGTTTACTGCAGGTTTCATAACTTCTTCCCGATT $\begin{array}{llllllllllllllllllllll}101 & V & H & N & E & W & Y & F & G & I & V & Y & C & R & F & H & N & F & F & P & I\end{array}$ 361 GCAGCTGTCTTCGCCAGTATTTACTCTATGACCGCTATAGCTCTGGACAGGTATATGGCG $\begin{array}{lllllllllllllllllllll}121 & A & A & V & F & A & S & I & Y & S & M & T & A & I & A & L & D & R & Y & M & A\end{array}$ 421 ATCATCCACCCGCTCCAGCAGCGTCTGTCGTCCGCTGAGACTAAACTGGTGGTGTGTGTG

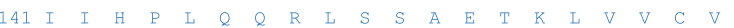
481 ATCTGGGCGCTGGCGCTAATGCTGGCGTTCCCTCAGTATTATTTCTCCAGCACGGCTCAG

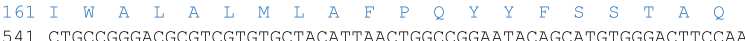

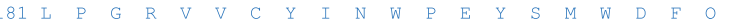
601 AAGACGTATTACGTCTGTGTGGTTGTGCTCATCTATTTTCTGCCTCTGCTGGTCATGGGT 201 K 661 TGTGCTTACCTGGTGGTGGGATGTTTTCTGTGGGCGAGCGAGATTCCTGGAGACTCTTCA

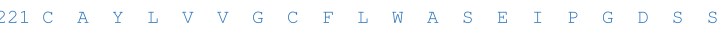
721 GATCGGTATCGAGAGCAGCTGATCGCCAAACGCAAGGTGGTGAAGATGATGATCGTCGTA $\begin{array}{lllllllllllllllllllll}241 & D & R & Y & R & E & \& & L & I & A & K & R & K & V & V & K & M & M & I & V & V \\ 7\end{array}$ 781 GTGTGCACCTTCGCCACCTGCTGGCTCCCCTATCATGTCTACTTCATAGTGCACCAGTT

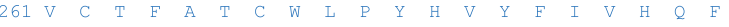
841 CTTCCACACTTGTTTGAGGAACGCTACATCCAGCAGGTCTACTTGGGGATAATGTGGCTA 281 L $P$ P 901 GCTATGAGCTCCACCATGTACAATCCCATCATCTACTGTCTTCTCAACGACAGGTTTCGA $\begin{array}{lllllllllllllllllllll}301 & \text { A } & \text { M } & \text { S } & \text { S } & \text { T } & \text { M } & \text { Y } & \text { N } & \text { P } & \text { I } & \text { I } & \text { Y } & \text { C } & \text { L } & \text { L } & N & D & R & F & R\end{array}$ $\begin{array}{llllllllllllllllllll}321 & A & G & F & Q & Q & A & F & S & W & C & P & C & V & P & Q & G & S & Y & E\end{array}$ 1021 CTGGAGCTCAAGTCTACTCGCTATCTTCAGACGCAGGCCAGCATCTTCAGGGCCAGCCGA

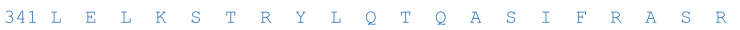
1081 ATGGAAACCACAGTATCCACAGTGATGCCAACTGGAGACGGAGAACTTCCAGAGAATCCA

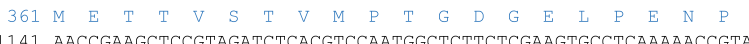
141 AACCGAAGCTCCGTAGATCTCACGTCCAATCGCICITCICCAAGTGCCTCAAAAACCGTA 201 TCCGAGTCCTCCTTCTATTCCAGTAACAACGTAGCATAGaaagaaacgtaggaagtatt 401 S E S F Y S S N N V A Stop tgtagtattattttagatgttagttttagtctttgtagtttcagtctaaagaatatctac tagctagtgagctccaaaacggtggcaaaatttgcatttagaagatataaacctgatata aacatctaaagtt.tgcagtttgcattttccgettaaatagatcaaggatctttttgatca

and reverse PCR primers are underlined in red. (C) PCR expression of tacr1a and tacr $1 b$. Full colour version of this figure available via http://dx.doi.org/ 10.1530/JME-12-0199.

Published by Bioscientifica Ltd 
$\mathrm{N}$-terminal

TM1

Human

Rat

Mouse

Zf Tacrla

Zf Tacrlb

Human

Rat

Mouse

Zf Tacrla

Zf Tacrlb

Human

Rat

Mouse

Zf Tacrla

Zf Tacrlb

Human

Rat

Mouse

Zf Tacrla

Zf Tacrlb

Human

Rat

Mouse

Zf Tacrla

Zf Tacrlb

Human

Rat

Mouse

Zf Tacrla

Zf Tacrlb

Human

Rat

Mouse

Zf Tacrla

Zf Tacrlb

Human

Rat

Mouse

Zf Tacrla

Zf Tacrlb
MDNVL PVDSDLSPNIISTMTISEP-------NOFVOPAWOIVLWAAAYTVIVVTSVVGNVVV 53 MDNVLPMDSDLFP(N)ISINTISES-------NQFVQPTWQIVLWAAAYTVIVVTSVVGNVVV 53 MDNVLPVDSDLFP(N)TSTISES-------NQFVQPTWQIVLWAAAYTVIVVTSVVGNVVV 53 MDS-FITSTDFPS(UNW(I)V(N)S(T)AQNETEIYWNQFVQPVWRIVLWAVAYSTIVVVSVVGNITV 59 MDPLYITSPNSSADNGSAGSPVNDTEVFGNQFVQPVWQIVLWAIAYCTIVLVSVVGNITV 60

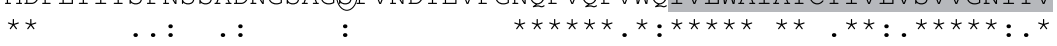

IL1

TM2

EL1

MWI I LAHKRMRTVTNYFLVNLAFAEASMAAFNTVVNFTYAVHNEWYYGLFYCKFHNFFPI 113 IWI I LAHKRMRTVDNYFLVNLAFAEACMAAFNTVVNF(T)YAVHNVWYYGLFYCKFHNFFPI 113 IWI I LAHKRMR(T) TNYFLVNLAFAEACMAAFNTVVNF(T)YAVHNVWYYGLFYCKFHNFFPI 113 IWI I LAHKRMR(T)VONYFLVNLAFAEASMSAFNTVINFVYSVHNEWYFGLYYCRFHNFFPI 119 IWI I LAHKRMRTVDNYFLVNLAFAEASMSAFNTV INFIYAVHNEWYFGLVYCRFHNFFPI 120

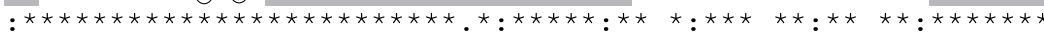

TM3 IL2

TM4

AAVFAS IYSMTAVAFDRYMAI I HPLQPRLSA(T)AT)KVVICVIWVLALLLAFPQGYYSTTET 173 AALFAS IYSMTAVAFDRYMA I HPLQPRLSA(T)ACKVVIFVIWVLALLLAFPQGYYSTTET 173 AALFAS IYSMTAVAFDRYMA I I HPLQPRL(SA(T)A(T)KVVIFVIWVLALLLAFPQGYYSTTET 173 AAVFAS IYSMTA IALDRYMA I I HPLQQRM(SA(T)OT)KVVIGVIWILALLLAFPQYYYSDTDQ 179 AAVFAS IYSMTA IALDRYMAI I HPLQQRL(S)SAET)KLVVCVIWALALMLAFPQYYFSSTAQ 180

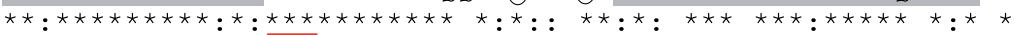

$$
\perp \quad \text { EL2 }
$$

TM5

IL3

MPSRVVCMIEWPEHP(NKIYEKVYHICVTVLIYFLPLLVIGYAYTVVGITLWASEIPGDSS 233 MPSRVVCMIEWPEHP(NRTYEKAYHICVTVLIYFLPLLVIGYAYTVVGITLWA(S)EIPGDSS 233 MPSRVVCMIEWPEHP(NRTYEKAYHICVTVLIYFLPLLVIGYAYTVVGITLWA(SEIPGDSSS 233 LPGRVVCYIDWPEYTLLDFKTMYFVCVAVLIYFLPLLVMGCAYLVVGLDLWA(S)E I PGD(SS) 239 LPGRVVCY INWPEYSMWDFQKTYYVCVVVLIYFLPLLVMGCAYLVVGCFLWASEIPGDSS 240

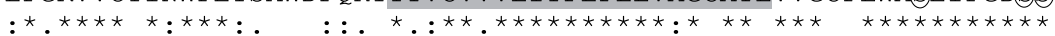

IL3

TM6

EL3

DRYHEQVSAKRKVVKMMIVVVCTFAICWLPFHIFFLLPYINPDLYLKKFIOOVYLAIMWL 293 DRYHEQVSAKRKVVKMMIVVVCTFAICWLPFHVFFLLPYINPDLYLKKFIQQVYLASMWL 293 DRYHEQVSAKRKVVKMMIVVVCTFAICWLPFHIFFLLPYINPDLYLKKFIQQVYLASMWL 293 DRYQEQL(TAKRKVVKMMIVVVCTFAVCWLPYHVYFLIHQFYPHLFEHTFIQQVYLTIMWL 299 DRYREQLIAKRKVVKMMIVVVCTFATCWLPYHVYFIVHQFLPHLFEERYIQQVYLGIMWL 300

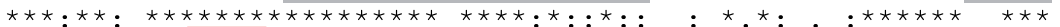

\section{TM7}

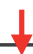

C-terminal

AMS STMYNPI IYCCLNDRFRLGFKHAFRCCPFI(S)AGDYEGLEMK(SPRYLQT)QGSVYKVISR 353 AMSSTMYNPI IYCCLNDRFRLGFKHAFRCCPFI(S)AGDYEGLEMK(ST)RYLQT)QSSVYKV(S)R 353 AMSSTMYNPI IYCCLNDRFRLGFKHAFRCCPFI(SAGDYEGLEMKSSTRYLQT)QS(S)VYKVSR 353 AMSSTMYNP I IYCCLNDRFRAGFKQAFRCCPCVPEGSYEGLELKSSTRYLOT)QT(S)LYRA(S)R 359 AMS STMYNPI IYCLLNDRFRAGFQQAFSWCPCVPQGSYEGLELK(ST)RYLQT)QASIFRA(S) 360

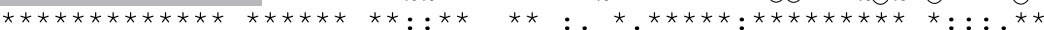

\section{C-terminal}

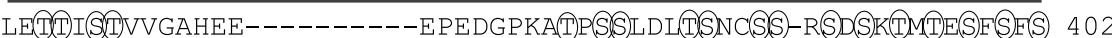

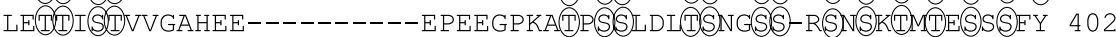

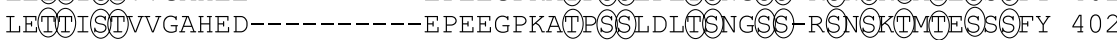
METSVISCVMQP SEDGHKVTFGSVRGAAVKPAARSPSREFTSNGSSSSR(S)I(S)K(T)V(S)E(T)S(S)FY 419 METLVSTVMPTGD--------- GELPENPNRS-SVDLTSNGSS-RSASKTVSEE-S(S)FY 406 $\cdot \star \star \cdot . \star \star *:$

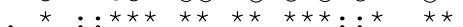

$\overline{(S)-N V I(S)-} 407$

(S)-NMLA - 407

(S)-NMLA- 407

(S)SNNLOE 426

(S)SNNVA- 412

$\star \star$. http://jme.endocrinology-journals.org DOI: 10.1530/JME-12-0199
(ㄷ) 2013 Society for Endocrinology Printed in Great Britain
Published by Bioscientifica Ltd. 
tacr1a (1281 bp; GenBank ID: 33455) and tacr1b (1239 bp; GenBank ID: 345672) (Fig. 1A, B and C). The ORFs of the tacr $1 a$ and tacr $1 b$ genes encoded proteins of 426 (predicted molecular weight of $48.96 \mathrm{kDa}$ ) and 412 amino acids (40.53 kDa) respectively (Fig. 2). Tacr1a and Tacr1b exhibited characteristic seven transmembrane domains (TMs), two consensus sites for $\mathrm{N}$-glycosylation sites for Tacr1a $\left(\mathrm{Asn}^{13}\right.$ and $\left.\mathrm{Asn}^{17}\right)$, and one possible site for Tacr1b $\left(\mathrm{Asn}^{14}\right)$ in the $\mathrm{N}$-terminal extracellular domain ( $\mathrm{N}$-terminal), together with two conserved Cys residues for Tacr1a $\left(\mathrm{Cys}^{111}\right.$ and $\mathrm{Cys}^{186}$ ) and for Tacr1b (Cys ${ }^{112}$ and Cys ${ }^{187}$ ), which can form a disulfide bond between the first and second extracellular loops. The NK receptors also showed putative phosphorylation sites on serine or threonine residues: in the N-terminal, in the first intracellular loop (IL1), in IL2, in IL3; in the carboxyl terminal domain (C-terminal), and a potential palmitoylation site on Cys residues for Tacr1a $\left(\mathrm{Cys}^{329}\right)$ and for Tacr1b $\left(\mathrm{Cys}^{330}\right)$ in the C-terminal domain. Moreover, Tacr1a and Tacr1b had a typical DRY motif at the border between TM3 and IL2 and a K/RK/RXXK/R moiety in IL3 (Fig. 2).

The Ensembl database (http://www.ensembl.org/) revealed that the tacr1a and tacr1b genes were found on chromosomes 5 and 11 respectively and formed by five exons. The zebrafish Tacr1a and Tacr1b homology with respect to human, rat, and mouse (TACR1) was $66 \%$ and when it was compared between them (Tacr1a and Tacr1b) it was $75 \%$.

\section{Phylogenetic analysis of zebrafish Tacr1a and Tacr1b}

A clear demarcation was found between zebrafish Tacr1s (Tacr1a and Tacr1b), Tacr2, and Tacr3, and their orthologs, supported by bootstrap values of 100,48 , and $99 \%(\mathrm{NJ}$; Fig. 3A) and 55, 70, and 94\% (ML; Fig. 3B). The zebrafish Tacr2 (predicted sequence) and Tacr3 (Zhou et al. 2012) amino acid sequences were used to check whether Tacr1a and Tacr $1 \mathrm{~b}$ could be aligned in Tacr 2 or Tacr 3 clusters and might not be duplicate genes. The phylogenetic analyses showed that Tacr1a and Tacr1b were aligned in the same cluster of Tacr1s, suggesting that Tacr1a and Tacr1b receptors are duplicate genes and paralogs of the Tacr2 and Tacr3 from zebrafish and orthologs of the TACR1, TACR2, and TACR3 of several species. We performed a double phylogenetic analysis using two different methods in order to corroborate that the replication of the alignment of the branches was indeed due to the divergence of the amino acid sequences, rather than to the phylogenetic algorithms employed. The zebrafish opioid receptors ZfMOR (NP_571782.1), ZfDOR1 (NP_571333.1), ZfDOR2 (NP_997920.1), and ZfKOR (NP_878306.1) were included as out-groups, as these and TACR1 belong to the GPCR superfamily (Herrero-Turrion \& Rodriguez 2008) and, additionally, both of them are involved in pain and analgesic processes respectively.

The following species (with their respective accession number) were employed to perform the phylogenetic analyses in the case of TAC1R: human (NP_001049.1), dog (NP_001012637.1), guinea pig (NP_001166332.1), rat (NP_036799.1), mouse (NP_033339.2), chicken (NP_990199.1), frog (NP_001106489.1; Xenopus tropicalis), fugu (AAQ02694), zebrafish Tacr1a (GenBank ID: 33455), and zebrafish Tacr1b (GenBank ID: 345672; also named Nk1a and Nk1b respectively); for TACR2: human (NP_001048.2), rat (NP_542946.1), mouse (NP_033340.2), and zebrafish Tacr2 (XP_001341981.1) (predicted sequence); in the case of TACR3: human (NP_001050.1), rat (NP_058749.1), mouse (NP_067357.1), and zebrafish Tacr3a1, Tacr3a2, and Tacr3b (Zhou et al. 2012).

\section{Temporal expression of tacr1a and tacr1b during embryogenesis}

In this study, the mRNA expression of tacr1a was gradually increased from 8 to $72 \mathrm{hpf}$ (Fig. 4A), except at $48 \mathrm{hpf}$, when tacr1a mRNA decreased with respect to $30 \mathrm{hpf}$. By contrast, the mRNA of tacr1b displayed a different expression pattern with respect to tacr1a (Fig. 4B). At early developmental stages, from 8 to $22 \mathrm{hpf}$, no marked changes were observed. When the CNS had been formed

\section{Figure 2}

Comparison of the deduced amino acid sequences of the TACR 1s from human, rat, mouse, and zebrafish (Zf). Asterisks, colons, and dots denote the identical amino acid, conserved substitutions, and semi-conserved substitutions respectively. Putative membrane-spanning sequences are highlighted in gray and are denoted by TM. N-terminal extracellular domain (N-terminal), carboxyl terminal domain (C-terminal), and IL and extracellular loops (EL) are indicated by horizontal lines above the sequences. The red circles indicate consensus $N$-linked glycosylation sites. The red line indicates the typical GPCR moieties, DRY and K/RK/RXXK/R. The black arrows denote the cysteine residues responsible for a disulphide bridge. The red arrow depicts a potential palmitoylation site. The black circles indicate potential intracellular serine and threonine phosphorylation sites. Full colour version of this figure available via http://dx.doi.org/ 10.1530/JME-12-0199. http://jme.endocrinology-journals.org DOI: 10.1530/JME-12-0199
C 2013 Society for Endocrinology Printed in Great Britain
Published by Bioscientifica Ltd 
A

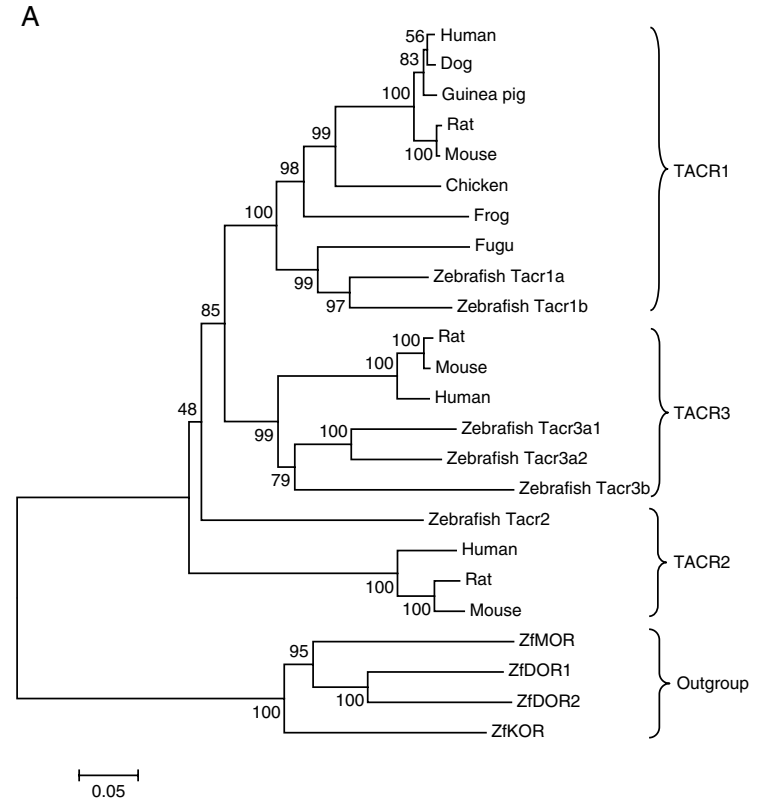

B

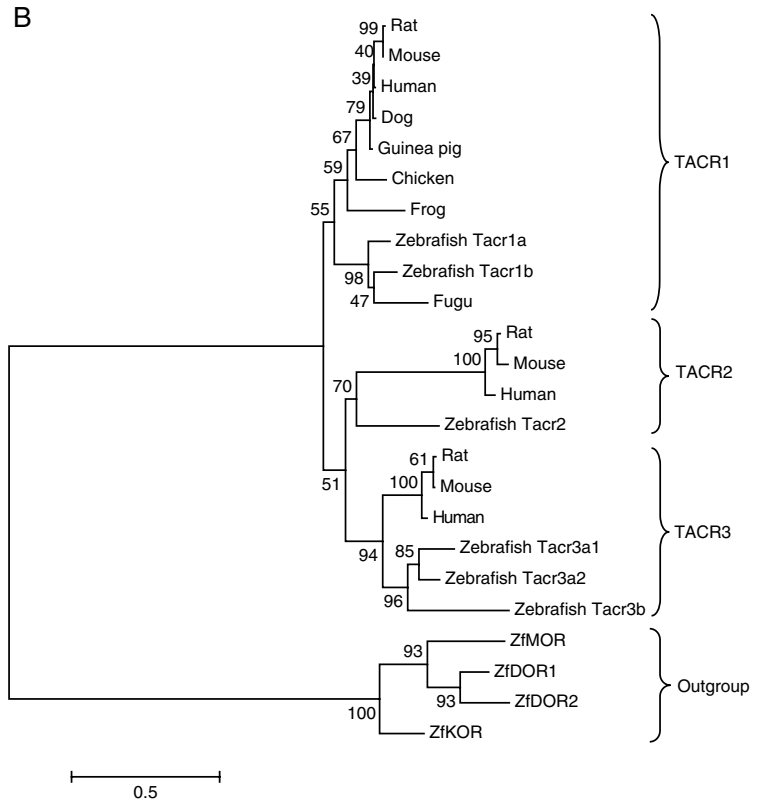

\section{Figure 3}

Phylogenetic analyses of vertebrate neurokinin receptors. The evolutionary history was inferred using the NJ (A) and ML (B) methods. The percentage of replicate trees in which the associated taxa clustered together in the

at $24 \mathrm{hpf}$, tacr $1 b$ showed a threefold increase with respect to $22 \mathrm{hpf}$. The expression of tacr $1 b$ decreased from 24 to $48 \mathrm{hpf}$. In addition, at $72 \mathrm{hpf}$, we observed that tacr $1 \mathrm{~b}$ reached the maximum peak of expression with respect to all the developmental stages studied (Fig. 4B).

The $8 \mathrm{hpf}$ stage was selected because at this moment, there is a reliable mRNA expression with no maternal interference. During the early stages of 16,19 , and $22 \mathrm{hpf}$, the segmentation and differentiation of the CNS are taking place and at $24 \mathrm{hpf}$, the zebrafish already have the five lobes (telencephalon, diencephalon, epiphysis, midbrain, and cerebellum) that conform the CNS. Organogenesis takes place between 24 and $48 \mathrm{hpf}$, and at $48 \mathrm{hpf}$, the morphogenesis of the primary organ systems has been completed. The end of embryonic development occurs at 72 hpf.

\section{tacr1a and tacr1b mRNA spatial expression at 24 and $48 \mathrm{hpf}$}

The tacr1a transcript was detected at $24 \mathrm{hpf}$ in different areas of the CNS (telencephalon, hypothalamus, diencephalon, midbrain, midbrain-hindbrainboundary (MHB), and hindbrain) and also peripherally in the somites (Fig. 5A and B). The expression of tacr $1 b$ at $24 \mathrm{hpf}$, like bootstrap test (1000 replicates) is shown next to the branches. The trees are drawn to scale, with branch lengths in the same units as those of the evolutionary distances used to infer the phylogenetic tree.

that of tacr1a, was found in the CNS (Fig. 5E and F; telencephalon, hypothalamus, diencephalon, MHB, midbrain, hindbrain, and spinal cord) and at the periphery (somites). At $48 \mathrm{hpf}$, tacr1a showed a similar pattern of expression in the CNS to that at $24 \mathrm{hpf}$ and it was also expressed in the medial longitudinal fasciculus (MLF). At the periphery, expression was less than at $24 \mathrm{hpf}$ but was observed in somites (Fig. 5C and D). tacr $1 b$ transcripts at the $48 \mathrm{hpf}$ stage (Fig. 5G and H) were also present in the CNS, similar to at $24 \mathrm{hpf}$, although with higher expression and with no expression in the spinal cord. At this stage, tacr $1 b$ was also expressed in the MLF and somites (Fig. 5G and $\mathrm{H}$; but less intensely than at $24 \mathrm{hpf}$ ).

\section{Effects of cocaine on the temporal expression of tacr1a and tacr1b}

Embryos exposed to cocaine (at $5 \mathrm{hpf}$ ) and analyzed at $24 \mathrm{hpf}$ showed a twofold increase in the expression of tacr1a with respect to the control group $(P<0.0001$; Fig. 6A). Also, the expression of tacr $1 b$ was increased by the actions of cocaine: threefold higher with respect to the control group ( $P=0.0194$; Fig. $6 \mathrm{~B})$. At $48 \mathrm{hpf}$, cocaine decreased the expression of tacr1a $(P=0.0006$; Fig. 6A). Conversely, cocaine led to a twofold increase in the

Published by Bioscientifica Ltd. 

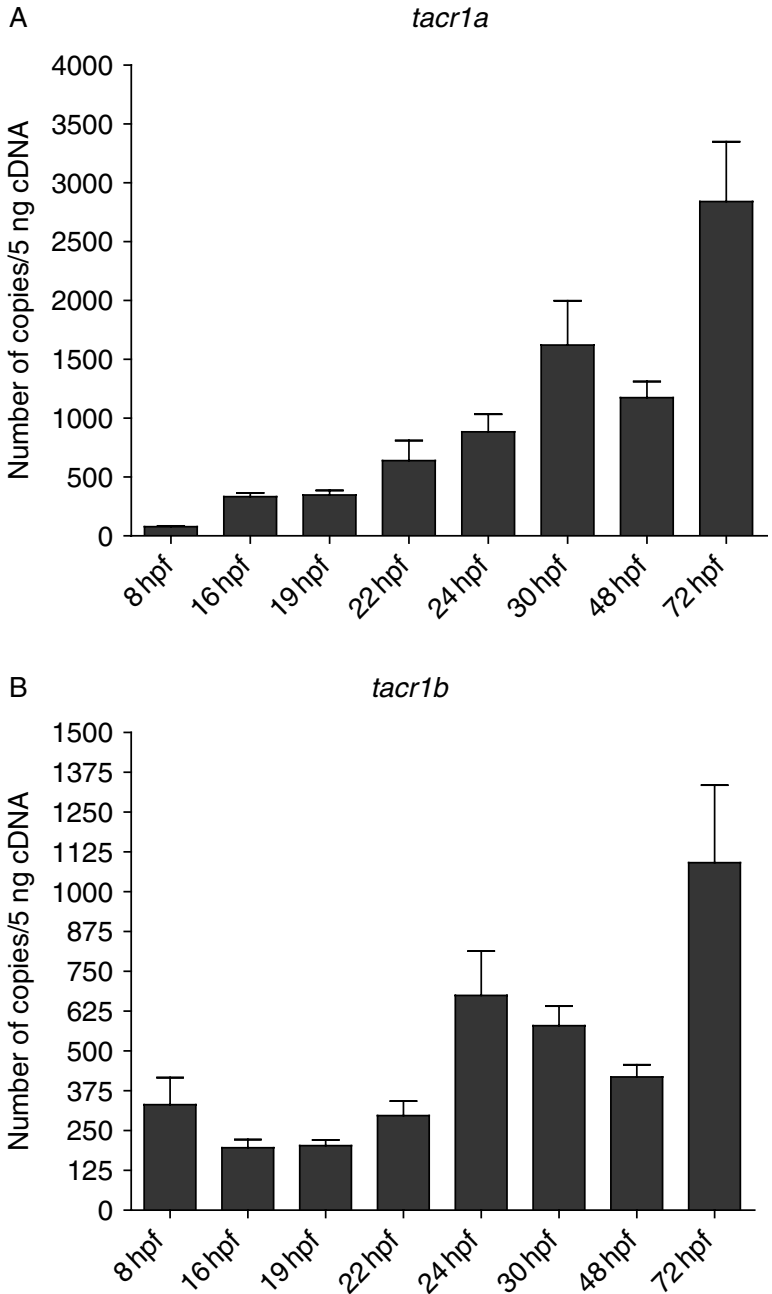

Figure 4

Expression of tacr1a (A) and tacr1b (B). qPCR analyses of transcript levels of neurokinin receptors were performed at several development stages: 8,16 , $19,22,24,30,48$, and $72 \mathrm{hpf}$. Gene expression levels were measured by fluorescence intensities using qPCR. The reference gene used to normalize the expression of the different genes studied by qPCR was ef1 $\alpha$ (NM_131263.1). Two hundred embryos were used to extract RNA and synthesize CDNA. Error bars represent means of mRNA copies at each developmental stage \pm s.E.M. Three independent experiments (each performed three times) were conducted for each stage.

expression levels of tacr1b $(P<0.0359$; Fig. 6B), in both cases with respect to the control group.

\section{Knockdown of ZfMOR, ZfDOR1, and ZfDOR2 and effects on tacr1a and tacr1b expression}

Knockdown of the ZfDOR2 opioid receptor with a MO elicited nearly eight times less expression of tacr $1 a$ at $48 \mathrm{hpf}$ with respect to the control group $(P=0.0010$; Fig. 7A) and the MO control group. Conversely, ZfMOR MO microinjection induced a fourfold increase in the expression of tacr $1 b(P<0.0001$; Fig. 7B) in comparison with the control and MO control groups. The ZfDOR1 and ZfDOR1/2 did not induce changes in the expression of any tacr1s.

\section{Effects of cocaine on the level of expression of tacr1a and} tacr1b transfected into HEK-293 cells

HEK-293 cells transfected with tacr1a and tacr1b coupled to pEGFP-C3 and pDsRed-N-1 respectively did not reveal morphological changes in tacr1 (Fig. 8A and B) and tacr $1 b$ (Fig. 8C and D) due to exposure to cocaine. Nevertheless, PCR results showed that cocaine decreased the expression levels of tacr1a (Fig. 8E) and tacr1b (Fig. 8F). This decrease, especially that of tacr1a, being noteworthy (Fig. 8E). We have also corroborated by qPCR that cocaine decreased the expression level of tacr1a dramatically $(P<0.0001$; Fig. 8I), and in the case of tacr $1 b$, the changes produced by cocaine were more apparent than those observed before in the PCR experiment (Fig. 8F), as cocaine elicited a reduction $(P<0.0001$; Fig. 8I). Before exposure to cocaine, we evaluated the toxic effect of cocaine on HEK-293 cells by microscopy observations and we did not find changes of cell viability in cocaine-treated and -untreated groups of cells. The doses of $0.5,1.5$, and $3 \mu \mathrm{M}$ were used and none produced toxicity in the cell culture. Moreover, we studied the expression of the reference gene $(G A P D H)$ by PCR and qPCR (Fig. 8A and B respectively), where this reference gene does not exhibit consistent changes. This indicates that the effects of cocaine altered the expression of tacr1 genes and is not related to the toxicity of cocaine on the HEK-293 cells.

\section{Discussion}

In this study, the tacr1a and tacr1b genes in zebrafish, which are duplicate genes of the TAC1R gene in mammals have been identified (Pennefather et al. 2004). The phylogenetic trees generated with the NJ and ML methods show that these receptors have a monophyletic origin, as the three TACRs derive from a common gene. In vertebrates, there are three TACRs (TACR1, TACR2, and TACR3; Pennefather et al. 2004) that were originated during evolution by duplications of a single ancestral receptor. In addition, both phylogenetic trees suggest that the TACRs (related to the pain process) were originated before the development of opioid receptors (analgesic receptors) during evolution, suggesting that during phylogenetic evolution first appeared pain and after its regulatory mechanism system.

Published by Bioscientifica Ltd. 

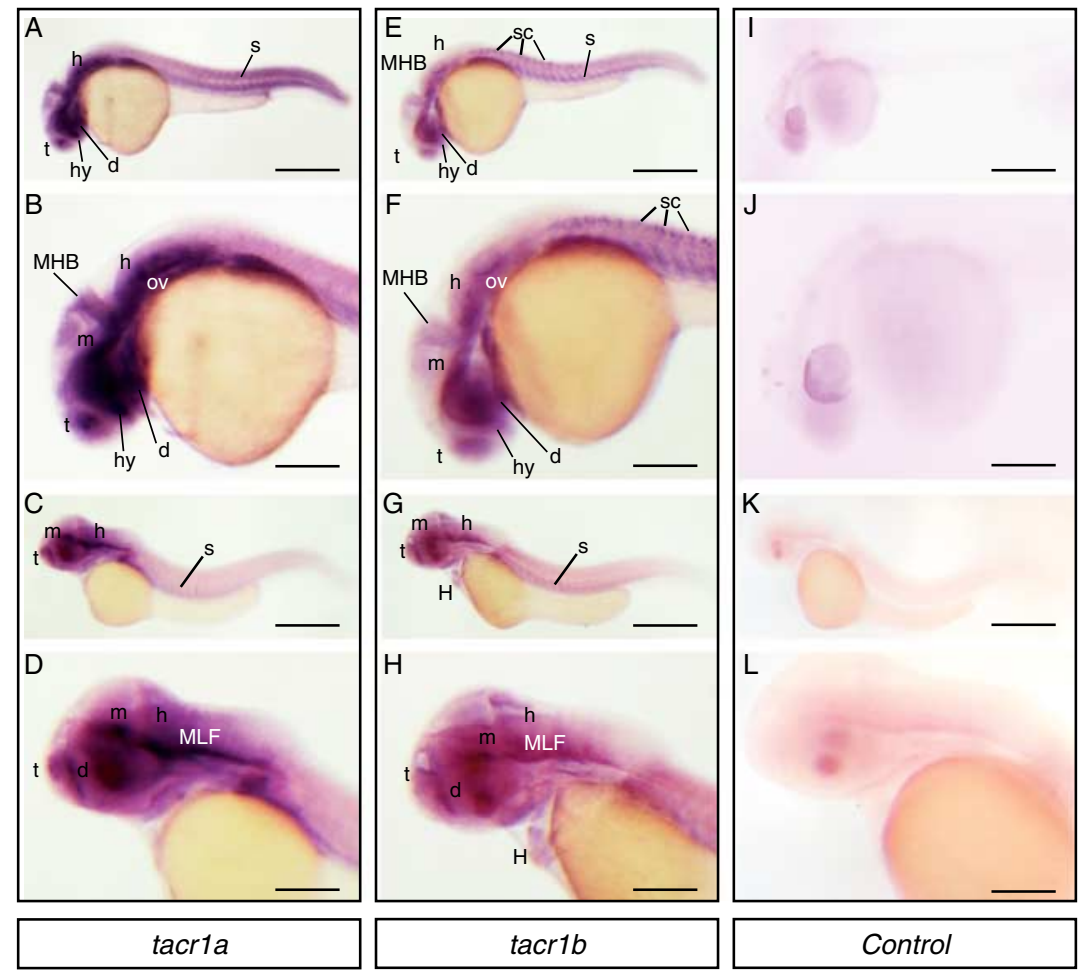

\section{Figure 5}

Distribution of tacr1a and tacr $1 \mathrm{~b}$ at 24 and $48 \mathrm{hpf}$ in embryos by wholemount ISH. tacr1a is present in the telencephalon, diencephalon, midbrainhindbrain boundary, hindbrain, and somites at $24 \mathrm{hpf}$ ( $\mathrm{A}$ and $\mathrm{B})$. tacr $1 \mathrm{~b}$ is present in telencephalon, diencephalon, midbrain, hindbrain, spinal cord, and somites at $24 \mathrm{hpf}$ ( $E$ and F). Lateral views (C and D) show that tacr1a is present in the telencephalon, diencephalon, midbrain, MLF, hindbrain, heart, and somites. In the case of $\operatorname{tacr1b}(\mathrm{G}$ and $\mathrm{H})$, it is present in the telencephalon, diencephalon, midbrain, MLF, hindbrain, spinal cord, and

Tacr1a and Tacr1b, displayed characteristics of the seven TM receptor, the two consensus sites for $N$-glycosylation sites in the human TAC1R (Asn ${ }^{14}$ and Asn ${ }^{18}$; Takeda et al. 1991) are conserved in zebrafish Tacr1a $\left(A s n^{13}\right.$ and $\left.A s n^{17}\right)$ but not in Tacr1b, where the asparagine residues are substituted by $\mathrm{Asp}^{14}$ and $\mathrm{Ala}^{18}$ (Fig. 2). This difference in the $\mathrm{N}$-glycosylation pattern between TACR1a and TACR1b could involve changes in the bioactivity of Tacr1b and affect its physical stability as the glycosylation of TACR1 may stabilize the receptor at the plasma membrane (Tansky et al. 2007). Two amino acid residues $-\mathrm{Val}^{116}$ and $\mathrm{Ile}^{290}$ - in the human TACR1 (Fong et al. 1992a) are conserved in zebrafish Tacr1a ( $\mathrm{Val}^{122}$ and $\mathrm{Ile}^{296}$ ) and Tacr1b (Val ${ }^{123}$ and Ile $^{297}$ ) receptors and are not present in the rat or mouse, where they are substituted by Leu ${ }^{116}$ and $\operatorname{Ser}^{290}$. These residues appear to be determinant for the binding of the selective non-peptide antagonist CP 96345 on the TACR1 type and they are also important in the Printed in Great Britain somites. Control group (I, J, K and L). All the embryos of the control group, from both tacr1a and tacr $1 b$, did not show staining; for this reason, we only included one control group. Scale bar, $250 \mu \mathrm{m} ; 6 \times$ magnification in $A, C, E$, $\mathrm{G}, \mathrm{I}$, and $\mathrm{K}$ and $12 \times$ magnification in $\mathrm{B}, \mathrm{D}, \mathrm{F}, \mathrm{H}, \mathrm{J}$, and $\mathrm{L}$. $t$, telencephalon; $\mathrm{d}$, diencephalon; hy, hypothalamus; $\mathrm{m}$, midbrain; MLF, medial longitudinal fasciculus; $h$, hindbrain; ov, otic vesicle; sc, spinal cord; $\mathrm{s}$, somites; $\mathrm{H}$, heart. The images obtained were documented with a fluorescent stereomicroscope (Leica M165 FC).

differentiation between species (Fong et al. 1992b). The replacement of $\mathrm{Val}^{116}$ and $\mathrm{Ile}^{290}$ of the human TACR1 by Leu $^{116}$ and Ser $^{290}$ in the rat confers sensitivity to the TACR1 antagonist RP 67 580. Likewise, a non-peptide TACR1 antagonist, WIN 51 708, binds to the rat TACR1type receptor but not to the human TACR1. This binding is mediated by Ser $^{290}$ in the rat TACR1 while human TACR1 has Ile ${ }^{290}$ (Venepalli et al. 1992). As zebrafish Tacr1a and Tacr1b have residues of isoleucine Ile $^{296}$ and $\mathrm{Ile}^{297}$ respectively; Fig. 2), this organism would be an alternative model in the study of TACR1 antagonist in the field of pain. Additionally, the potential putative phosphorylation sites on serine or threonine residues in zebrafish Tacr1a and Tacr1b show that these receptors may be able to shed new light on the desensitization and internalization of TACR1. It is controversial if fishes feel pain, but during the last decade, important evidence has been shown concerning the fact that fishes can perceive pain.

Published by Bioscientifica Ltd. 


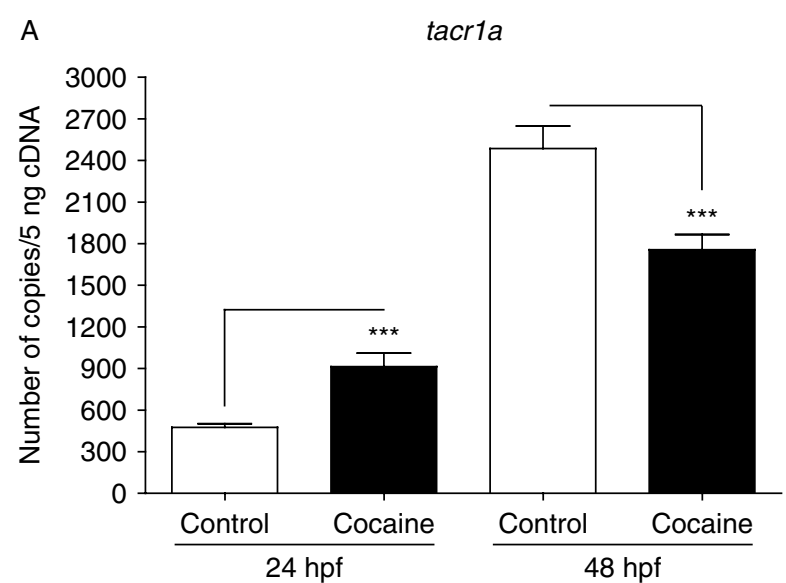

B tacr1b

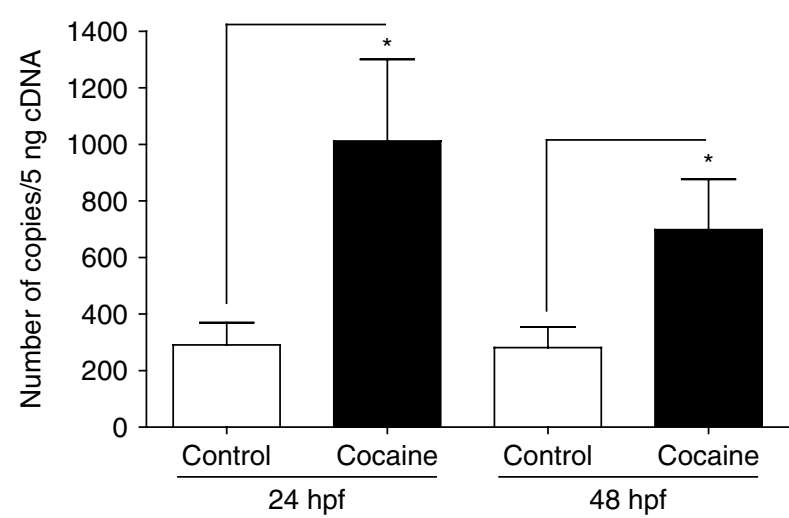

Figure 6

Effects of cocaine on the expression levels of tacr1a (A) and tacr1b (B) at 24 and $48 \mathrm{hpf}$. Two hundred embryos were used to extract RNA and synthesize CDNA. Error bars represent means of mRNA copies at each developmental stage \pm s.E.M. Three independent experiments (each carried out three times) were performed for each stage. $P$ values were calculated by a two-tailed unpaired Student's $t$-test: $* P<0.05$ and $* * * P<0.001$.

In this sense, the goldfish (Carassius auratus) and rainbow trout (Oncorhynchus mykiss) have been reported to present nociceptors that respond to mechanical, thermal, and chemical stimulations (Sneddon 2003a, Dunlop \& Laming 2005, Ashley et al. 2007) and morphine reduced dramatically the behavioral and physiological responses after injection of a noxious stimulus (Sneddon 2003b). As a teleost fish, zebrafish, has an analgesic system, opioid system (Gonzalez-Nunez \& Rodriguez 2009), and according to our findings, this organism also has the receptor of SP, Tacr1a and Tacr1b. It can be deduced that this organism through this nociceptive and tachykinin system can feel pain. Unfortunately, at present, a prototypic behavioral pain model in zebrafish has not been developed, as in mouse and rat, to corroborate the molecular and electrophysiological findings of pain in fishes (Sneddon 2003a, Dunlop \& Laming 2005, Ashley et al. 2007). However, all the recent studies using the zebrafish as a model point to this organism as a new potential model in the study of pain.

One interesting finding of our work is that the expression of tacr1a (Fig. 4A) is increasing since the early zebrafish stages of development ( $8 \mathrm{hpf}$, gastrulation) until the end of the CNS development ( $\sim 24 \mathrm{hpf})$, primary organogenesis (48 hpf), and embryogenesis (72 hpf), suggesting that these receptors are involved in the formation of the CNS, organogenesis, and embryogenesis in general. This fact is in agreement with studies that SP, ligand of the TACR1, is increasing in number during embryonic development of the CNS (Gilbert \& Emson 1979). Moreover, it has been reported that TAC1R could be important in the development of motorneurons in the rat during embryonic development (St John \& Stephens 1992, St John et al. 1997). One characteristic shared between tacr $1 a$ and tacr $1 b$ is that both are expressed at high levels at $72 \mathrm{hpf}$, although the expression of tacr $1 \mathrm{a}$ is almost threefold higher with respect to tacr1b (Fig. 4A and B). Also, during several embryonic stages of development, tacr $1 a$ mRNA expression was greater than tacr $1 b$. This fact suggests that tacr1a could have a major role, during zebrafish embryogenesis, in comparison to tacr $1 b$, as a subfunctionalization of the gene can occur after a gene duplication event (Postlethwait 2007).

In zebrafish, tacr1a and tacr1b (Fig. 5) were mainly expressed at $24 \mathrm{hpf}$ in the CNS (telencephalon, midbrain, diencephalon, hypothalamus, and hindbrain) and the peripheral (nervous system somites), with the difference that tacr $1 b$ was also expressed in the spinal cord. The expression of tacr $1 b$ in the spinal cord is consistent with the findings of TACR1 (mRNA) expression in the rat spinal cord (McCarson \& Krause 1994, 1996, McCarson 1999, Todd et al. 2002, Gamboa-Esteves et al. 2004), suggesting that in the spinal cord tacr $1 b$ might play a major role in comparison with tacr1a.

There is evidence of interaction of DOR/PPTA, as it has been reported that DOR coexist with PPTA, in the rat dorsal root ganglia in the primary afferent neurons (Minami et al. 1995) and PPTA can modulate the sensitivity of nociceptive afferents to opioids (Guan et al. 2005). In the present work, we report that knockdown of ZfDOR2 downregulates the expression of tacr1a, which suggests that DOR/TAC1R can have an important role during embryonic development and also in pain modulation (Minami et al. 1995, Guan et al. 2005). The difference of our work in relation to Minami et al. (1995) and Guan et al. (2005) is that we studied the mRNA

Published by Bioscientifica Ltd. 
A

tacr1a

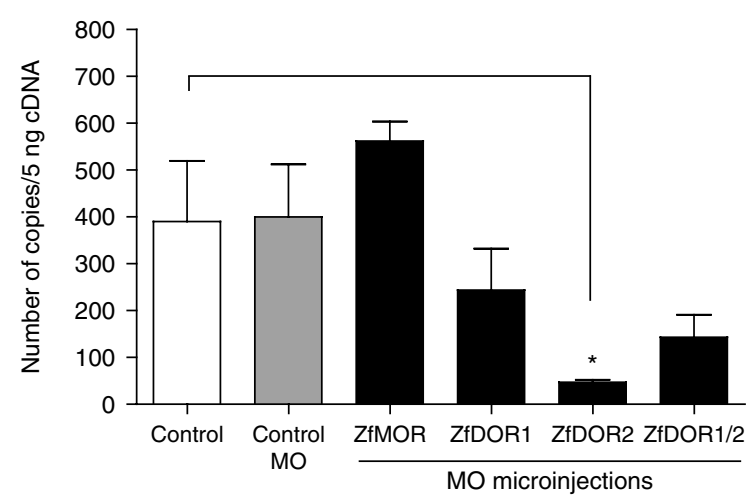

B

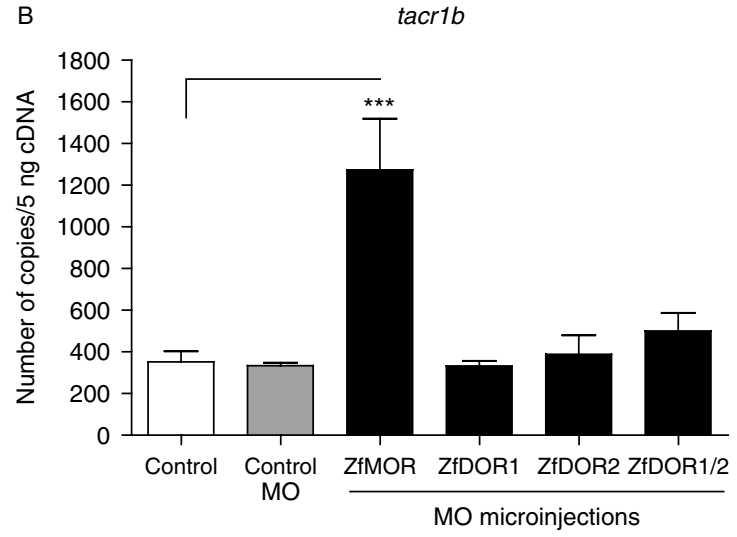

Figure 7

Effects of MOs on the expression levels of tacr1a (A) and tacr1b (B) at $48 \mathrm{hpf}$. MOs of ZfMOR, ZfDOR1, ZfDOR2, and ZfDOR1-ZfDOR2 (ZfDOR1/2) were microinjected into the yolk in the one-to four-cell stage. Two hundred embryos were used for RNA extraction. Each bar represents the number of copies of CDNA \pm s.E.M. For each stage, three experiments were performed, each three times. Between-group differences in $s p$, tacr $1 a$, and tacr $1 b$ levels were analyzed using ANOVA with the post hoc Dunnett's test: $* P<0.05$ and $* * * P<0.001$.

expression (of Tacr1a and Tacr1b) in whole-mount zebrafish embryos in one stage of embryonic development (48 hpf) while the other studies were done in adult rats and mice. Also, several studies have described that TACR1/MOR functionally interact and mediate the regulation of MOR-TACR1 trafficking, where the activation of TACR1 by SP can induce or inhibit the endocytosis of MOR (Pfeiffer et al. 2003, Yu et al. 2009). Our results show that downregulation of ZfMOR increases the expression of tacr $1 b$, suggesting that MOR could be positively regulating TACR1. According to our results, a possible mechanism of ZfDOR2 and ZfMOR antisense knockdown leading to changes in expression of tacr1a and tacr $1 b$ could be explained as an interaction of these receptors in the cell membrane or gene expression, but these postulates have to corroborate with future studies.
The importance of our research is that the tachykinin system and the opioid receptors are closely related, where changes induced in the tachykinin system could modify the functions of opioid receptors. As DOR and MOR interact (in the cell membrane) and DOR modulates the function of MOR (Gomes et al. 2004), TACRs (via MOR or indirectly via DOR) would modulate the activity of the opioid receptors. In this study, the knockdown of ZfDOR2 and ZfMOR regulated the expression of tacr $1 a$ and tacr $1 b$ respectively, indicating that tachykinin and the opioid systems are closely interrelated in zebrafish, as has been described in mammals (Minami et al. 1995, Pfeiffer et al. 2003, Guan et al. 2005, Yu et al. 2009). As the set of MOs of opioid receptors was effective, as previously demonstrated by Sanchez-Simon et al. (2010), and replicated in our study, we are convinced that the changes on the expression of the TACRs are a consequence of the downregulation of the opioid receptors. As we are studying the changes of gene expression in vivo whole-mount zebrafish embryos, it is difficult to know whether the effects that we observe are specifically produced by opioid receptors as other genes or elements can also regulate both the opioid and the tachykinin system.

SP is considered a neurotransmitter of the primary sensory neurons that mediate the synaptic transmission of pain (Otsuka \& Yoshioka 1993, Seybold 2009), which means that changes observed by cocaine actions in the mRNA expression of tacr1a and tacr1b (Fig. 6A and B respectively) could affect pain processes and alterations in the development of pain and hyperalgesia (Stanley \& Safford 2004) such as in embryogenesis. Conversely, our results show that cocaine exerts different actions in human cells and in zebrafish embryos, probably because in whole organisms cocaine must cross different barriers before exerting its actions on its targets, and also, it should be considered that it can be metabolized. As cocaine presents a high liposolubility, this drug rapidly passes through the cell membrane by passive transport (Schenker et al. 1993); this could explain the alterations of the expression of the $\operatorname{tacr} 1 a / b$ genes transfected in the HEK-293 cells. It would be interesting to study the amount of cocaine that is passing through the HEK-293 cell membrane. Moreover, according to our previous study, $\sim 12 \% 1.5 \mu \mathrm{M}$ cocaine is passing the chorion of zebrafish embryos (R López-Bellido, K Barreto-Valer, FM SanchezSimon \& RE Rodriguez 2012, unpublished observations) and up to $80 \%$ the placenta (Schenker et al. 1993). Thus, as we found that $1.5 \mu \mathrm{M}$ cocaine did not induce toxicity in the HEK-293 cells and produced no evident changes in the expression of tacr $1 a$ and $\operatorname{tacr} 1 b$ and the reference gene

Published by Bioscientifica Ltd 

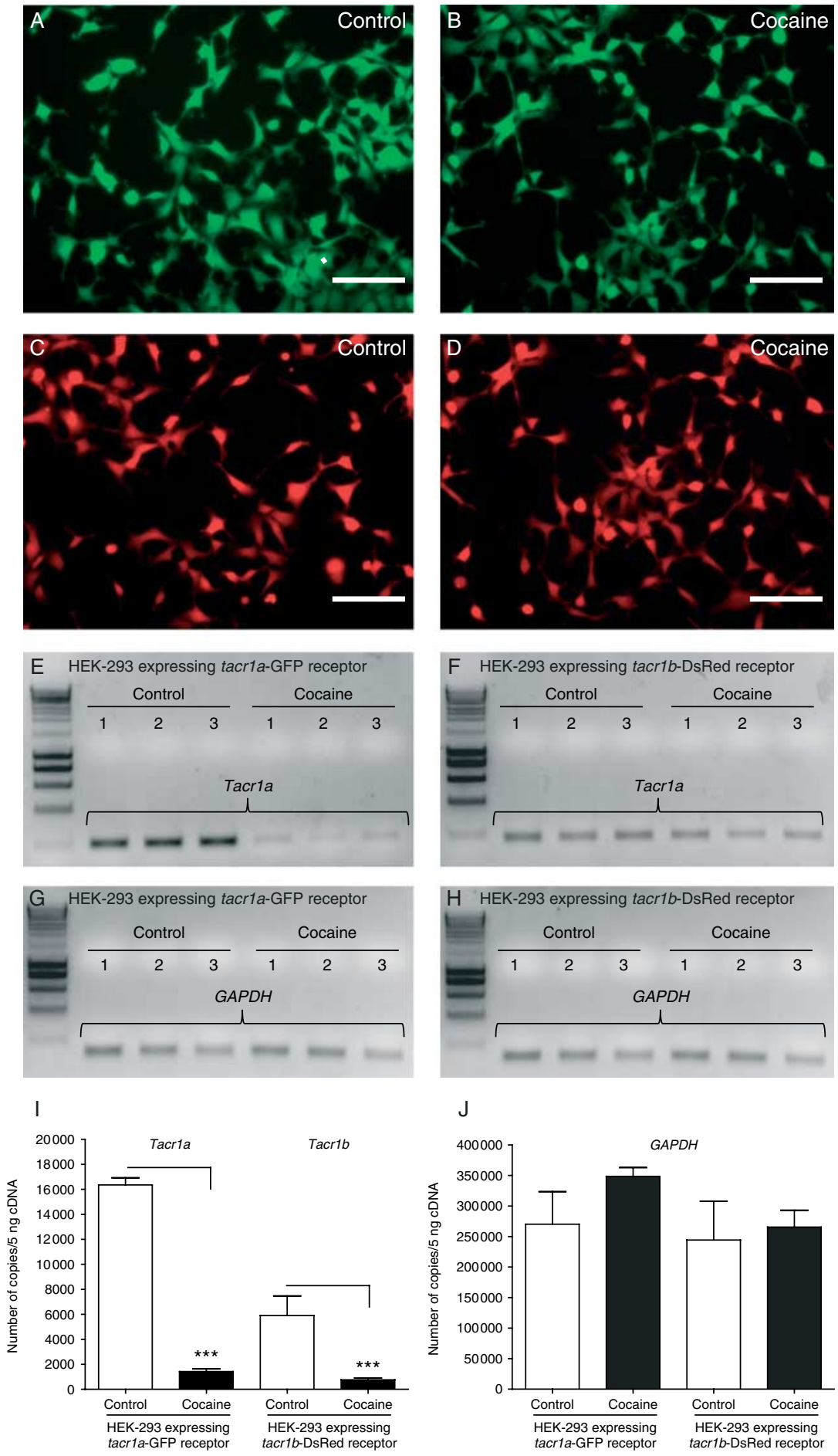

Figure 8

HEK-293 cells expressing tacr1a and tacr $1 b$ from zebrafish. HEK-293 cells were transfected with tacr1a-GFP receptor (A and B) and tacr1b-DsRed receptor $(C$ and $D)$. The pictures on the left and on the right correspond to the control ( $A$ and $C$ ) and cocaine groups ( $B$ and $D$ ) respectively. Scale bar, $100 \mu \mathrm{M}$. The pictures were taken using epifluorescent microscope (Eclipse TE2000-S, Nikon). Cocaine induced a decrease in tacr1a (E) and tacr1b (F), GAPDH was

used as a reference gene for both tacr1a $(\mathrm{G})$ and tacr1b $(\mathrm{H})$ expression in the $P C R$ results. Effects of cocaine on the expression of tacr1a and tacr $1 b$ (I and $J$ ) analyzed by qPCR. Cocaine induced a decrease in tacr1a and tacr1b expression (I), GAPDH was used as a reference gene (J). Three independent experiments, each performed three times, were carried out. $P$ values were calculated by unpaired two-tailed Student's $t$-test: $* * * P<0.001$.

Published by Bioscientifica Ltd. 
$G A P D H$, we believe that changes observed in the expression of tacr1s are due to the action of cocaine. HEK-293 cells do not express the dopamine transporter (DAT; Storch et al. 1999), and for this reason, we believe that cocaine is not working via DAT.

In conclusion, the tacr1s from zebrafish, tacr1a and tacr $1 b$, are the product of a gene that became duplicated during evolution. The expression of tacr1a and tacr1b mRNAs in the CNS and at the periphery during the embryonic stages of development shows that the tachykinin system could exert important actions in embryogenesis in zebrafish. Also, opioid receptors can regulate the expression of tacr $1 a$ and tacr $1 b$, which indicates that the opioid and tachykinin systems are tightly linked. Finally, the influence of cocaine on the expression level of tacr $1 a$ and tacr $1 b$ could affect embryogenesis in zebrafish.

\section{Declaration of interest}

The authors declare that there is no conflict of interest that could be perceived as prejudicing the impartiality of the research reported.

\section{Funding}

This work was supported by the Spanish Ministry of Education and Science (Grant SAF2010-18597) and the Regional Government of Castilla y León (Grant B1039/SA25/10). R L-B was supported by a grant, for his PhD studies, from the Spanish Ministry of Foreign Affairs, and the Agency for International Development Cooperation (MAEC-AECID).

\section{Author contribution statement}

$R \quad L-B$ and $R E R$ conceived and designed the experiments. $R$ L-B performed the experiments. R L-B and R E R analyzed the data. R L-B and $K$ B-V contributed reagents/materials/analysis tools. $R$ L-B and $R E$ wrote the manuscript.

\section{Acknowledgements}

The authors thank Dr Luis Fernando Alguacil for kindly providing the cocaine $\mathrm{HCl}$ that has been used in this work and Dr Manuel J Herrero Turrion for useful suggestions on the phylogenetic analyses.

\section{References}

Aicher SA, Punnoose A \& Goldberg A $2000 a \mu$-Opioid receptors often colocalize with the substance P receptor (NK1) in the trigeminal dorsal horn. Journal of Neuroscience 20 4345-4354.

Aicher SA, Sharma S, Cheng PY, Liu-Chen LY \& Pickel VM $2000 b$ Dual ultrastructural localization of $\mu$-opiate receptors and substance $P$ in the dorsal horn. Synapse 36 12-20. (doi:10.1002/(SICI)1098-2396 (200004)36:1<12::AID-SYN2 > 3.0.CO;2-E)

Ashley PJ, Sneddon LU \& McCrohan CR 2007 Nociception in fish: stimulus-response properties of receptors on the head of trout Oncorhynchus mykiss. Brain Research 1166 47-54. (doi:10.1016/ j.brainres.2007.07.011)
Chakraborty C, Hsu CH, Wen ZH, Lin CS \& Agoramoorthy G 2009 Zebrafish: a complete animal model for in vivo drug discovery and development. Current Drug Metabolism 10 116-124. (doi:10.2174/ 138920009787522197)

Commons KG 2010 Neuronal pathways linking substance P to drug addiction and stress. Brain Research 1314 175-182. (doi:10.1016/ j.brainres.2009.11.014)

Dempsey D, Jacob P III, Partridge JC, Jones RT, Panganiban K \& Rowbotham MC 1999 Cocaine metabolite kinetics in the newborn. Journal of Analytical Toxicology 23 24-28.

DeVane CL 2001 Substance P: a new era, a new role. Pharmacotherapy 21 1061-1069. (doi:10.1592/phco.21.13.1061.34612)

Dunlop R \& Laming P 2005 Mechanoreceptive and nociceptive responses in the central nervous system of goldfish (Carassius auratus) and trout (Oncorhynchus mykiss). Journal of Pain 6 561-568. (doi:10.1016/j.jpain. 2005.02.010)

Fong TM, Yu H, Huang RR \& Strader CD 1992a The extracellular domain of the neurokinin-1 receptor is required for high-affinity binding of peptides. Biochemistry 31 11806-11811. (doi:10.1021/bi00162a019)

Fong TM, Yu H \& Strader CD $1992 b$ Molecular basis for the species selectivity of the neurokinin-1 receptor antagonists CP-96,345 and RP67580. Journal of Biological Chemistry 267 25668-25671.

Gadd CA, Murtra P, De Felipe C \& Hunt SP 2003 Neurokinin-1 receptorexpressing neurons in the amygdala modulate morphine reward and anxiety behaviors in the mouse. Journal of Neuroscience 23 8271-8280.

Gamboa-Esteves FO, McWilliam PN \& Batten TF 2004 Substance P (NK1) and somatostatin (sst2A) receptor immunoreactivity in NTS-projecting rat dorsal horn neurones activated by nociceptive afferent input. Journal of Chemical Neuroanatomy 27 251-266. (doi:10.1016/j.jchemneu.2004.04.001)

Garzón M \& Pickel VM 2001 Plasmalemmal $\mu$-opioid receptor distribution mainly in nondopaminergic neurons in the rat ventral tegmental area. Synapse 41 311-328. (doi:10.1002/syn.1088)

Gilbert RF \& Emson PC 1979 Substance P in rat CNS and duodenum during development. Brain Research 171 166-170. (doi:10.1016/00068993(79)90745-5)

Gomes I, Gupta A, Filipovska J, Szeto HH, Pintar JE \& Devi LA 2004 A role for heterodimerization of mu and delta opiate receptors in enhancing morphine analgesia. PNAS 101 5135-5139. (doi:10.1073/pnas. 0307601101)

Gonzalez-Nunez V \& Rodriguez RE 2009 The zebrafish: a model to study the endogenous mechanisms of pain. ILAR Journal 50 373-386.

Guan JS, Xu ZZ, Gao H, He SQ, Ma GQ, Sun T, Wang LH, Zhang ZN, Lena I, Kitchen I et al. 2005 Interaction with vesicle luminal protachykinin regulates surface expression of $\delta$-opioid receptors and opioid analgesia. Cell 122 619-631. (doi:10.1016/j.cell.2005.06.010)

Harrison S \& Geppetti P 2001 Substance P. International Journal of Biochemistry \& Cell Biology 33 555-576. (doi:10.1016/S13572725(01)00031-0)

Herrero-Turrion MJ \& Rodriguez RE 2008 Bioinformatic analysis of the origin, sequence and diversification of $\mu$ opioid receptors in vertebrates. Molecular Phylogenetics and Evolution 49 877-892. (doi:10.1016/j.ympev. 2008.09.016)

Kombian SB, Ananthalakshmi KV, Zidichouski JA \& Saleh TM 2009 Substance $P$ and cocaine employ convergent mechanisms to depress excitatory synaptic transmission in the rat nucleus accumbens in vitro. European Journal of Neuroscience 29 1579-1587. (doi:10.1111/j.14609568.2009.06704.x)

Laurent V, Leung B, Maidment N \& Balleine BW $2012 \mu$ - and $\delta$-Opioidrelated processes in the accumbens core and shell differentially mediate the influence of reward-guided and stimulus-guided decisions on choice. Journal of Neuroscience 32 1875-1883. (doi:10.1523/JNEUROSCI.4688-11.2012)

Lohi O, Parikka M \& Ramet M 2012 The zebrafish as a model for paediatric diseases. Acta Paediatrica. In press. (doi:10.1111/j.1651-2227.2012. 02835.x)

Published by Bioscientifica Ltd. 
Maeno H, Kiyama H \& Tohyama M 1993 Distribution of the substance P receptor (NK-1 receptor) in the central nervous system. Brain Research. Molecular Brain Research 18 43-58. (doi:10.1016/0169-328X(93)90172-L)

Maggi CA 1995 The mammalian tachykinin receptors. General Pharmacology 26 911-944.

Maggi CA 2000 The troubled story of tachykinins and neurokinins. Trends in Pharmacological Sciences 21 173-175. (doi:10.1016/S0165-6147(00)01463-2)

Matthes HW, Maldonado R, Simonin F, Valverde O, Slowe S, Kitchen I, Befort K, Dierich A, Le Meur M, Dolle P et al. 1996 Loss of morphineinduced analgesia, reward effect and withdrawal symptoms in mice lacking the $\mu$-opioid-receptor gene. Nature 383 819-823. (doi:10.1038/ 383819a0)

McCarson KE 1999 Central and peripheral expression of neurokinin-1 and neurokinin-3 receptor and substance P-encoding messenger RNAs: peripheral regulation during formalin-induced inflammation and lack of neurokinin receptor expression in primary afferent sensory neurons. Neuroscience 93 361-370. (doi:10.1016/S0306-4522(99)00102-5)

McCarson KE \& Krause JE 1994 NK-1 and NK-3 type tachykinin receptor mRNA expression in the rat spinal cord dorsal horn is increased during adjuvant or formalin-induced nociception. Journal of Neuroscience $\mathbf{1 4}$ 712-720.

McCarson KE \& Krause JE 1996 The neurokinin-1 receptor antagonist LY306,740 blocks nociception-induced increases in dorsal horn neurokinin-1 receptor gene expression. Molecular Pharmacology 50 1189-1199.

Minami M, Maekawa K, Yabuuchi K \& Satoh M 1995 Double in situ hybridization study on coexistence of $\mu$-, $\delta$ - and $\kappa$-opioid receptor mRNAs with preprotachykinin A mRNA in the rat dorsal root ganglia. Brain Research. Molecular Brain Research 30 203-210. (doi:10.1016/0169328X(94)00290-U)

Nakaya Y, Kaneko T, Shigemoto R, Nakanishi S \& Mizuno N 1994 Immunohistochemical localization of substance P receptor in the central nervous system of the adult rat. Journal of Comparative Neurology 347 249-274. (doi:10.1002/cne.903470208)

Ogawa S, Ramadasan PN, Goschorska M, Anantharajah A, Ng KW \& Parhar IS 2012 Cloning and expression of tachykinins and their association with kisspeptins in the brains of zebrafish. Journal of Comparative Neurology 520 2991-3012. (doi:10.1002/cne.23103)

Otsuka M \& Yoshioka K 1993 Neurotransmitter functions of mammalian tachykinins. Physiological Reviews 73 229-308.

Pennefather JN, Lecci A, Candenas ML, Patak E, Pinto FM \& Maggi CA 2004 Tachykinins and tachykinin receptors: a growing family. Life Sciences 74 1445-1463. (doi:10.1016/j.lfs.2003.09.039)

Pfeiffer M, Kirscht S, Stumm R, Koch T, Wu D, Laugsch M, Schroder H, Hollt V $\&$ Schulz S 2003 Heterodimerization of substance $P$ and $\mu$-opioid receptors regulates receptor trafficking and resensitization. Journal of Biological Chemistry 278 51630-51637. (doi:10.1074/jbc.M307095200)

Pickel VM, Douglas J, Chan J, Gamp PD \& Bunnett NW 2000 Neurokinin 1 receptor distribution in cholinergic neurons and targets of substance $P$ terminals in the rat nucleus accumbens. Journal of Comparative Neurology 423 500-511. (doi:10.1002/1096-9861(20000731)423:3< 500::AID-CNE12>3.0.CO;2-9)

Postlethwait JH 2007 The zebrafish genome in context: ohnologs gone missing. Journal of Experimental Zoology. Part B, Molecular and Developmental Evolution 308 563-577. (doi:10.1002/jez.b.21137)

Regoli D, Drapeau G, Dion S \& D'Orleans-Juste P 1987 Pharmacological receptors for substance P and neurokinins. Life Sciences 40 109-117. (doi:10.1016/0024-3205(87)90349-3)

Regoli D, Boudon A \& Fauchere JL 1994 Receptors and antagonists for substance P and related peptides. Pharmacological Reviews 46 551-599.

Sanchez-Simon FM, Zhang XX, Loh HH, Law PY \& Rodriguez RE 2010 Morphine regulates dopaminergic neuron differentiation via miR-133b. Molecular Pharmacology 78 935-942. (doi:10.1124/mol.110.066837)
Santoriello C \& Zon LI 2012 Hooked! Modeling human disease in zebrafish. Journal of Clinical Investigation 122 2337-2343. (doi:10.1172/JCI60434) Schenker S, Yang Y, Johnson RF, Downing JW, Schenken RS, Henderson GI $\&$ King TS 1993 The transfer of cocaine and its metabolites across the term human placenta. Clinical Pharmacology and Therapeutics $\mathbf{5 3}$ 329-339. (doi:10.1038/clpt.1993.29)

Seybold VS 2009 The role of peptides in central sensitization. Handbook of Experimental Pharmacology 451-491.

Shults CW, Quirion R, Chronwall B, Chase TN \& O'Donohue TL 1984 A comparison of the anatomical distribution of substance $P$ and substance $\mathrm{P}$ receptors in the rat central nervous system. Peptides 5 1097-1128. (doi:10.1016/0196-9781(84)90177-3)

Sneddon LU 2003a Trigeminal somatosensory innervation of the head of a teleost fish with particular reference to nociception. Brain Research 972 44-52. (doi:10.1016/S0006-8993(03)02483-1)

Sneddon LU 2003 $b$ The evidence for pain in fish: the use of morphine as an analgesic. Applied Animal Behaviour Science 83 153-162. (doi:10.1016/ S0168-1591(03)00113-8)

Stanley AH \& Safford MM 2004 Treating chronic pain in the presence of substance abuse. Journal of the National Medical Association 961102-1104.

St John PA \& Stephens SL 1992 Development of substance P receptors on rat motoneurons in vitro. Developmental Biology 151 154-165. (doi:10.1016/0012-1606(92)90223-4)

St John PA, Ludwig CP \& Lai J 1997 Substance P receptor expression and cellular responses to substance $P$ in prenatal rat spinal cord cells. Journal of Receptors and Signal Transduction Research 17 569-583. (doi:10.3109/10799899709039150)

Storch A, Ludolph AC \& Schwarz J 1999 HEK-293 cells expressing the human dopamine transporter are susceptible to low concentrations of 1-methyl-4-phenylpyridine (MPP+) via impairment of energy metabolism. Neurochemistry International 35 393-403. (doi:10.1016/ S0197-0186(99)00083-2)

Takeda Y, Chou KB, Takeda J, Sachais BS \& Krause JE 1991 Molecular cloning, structural characterization and functional expression of the human substance P receptor. Biochemical and Biophysical Research Communications 179 1232-1240. (doi:10.1016/0006-291X(91)91704-G)

Tansky MF, Pothoulakis C \& Leeman SE 2007 Functional consequences of alteration of $N$-linked glycosylation sites on the neurokinin 1 receptor. PNAS 104 10691-10696. (doi:10.1073/pnas.0703394104)

Todd AJ, Puskar Z, Spike RC, Hughes C, Watt C \& Forrest L 2002 Projection neurons in lamina I of rat spinal cord with the neurokinin 1 receptor are selectively innervated by substance P-containing afferents and respond to noxious stimulation. Journal of Neuroscience 22 4103-4113.

Venepalli BR, Aimone LD, Appell KC, Bell MR, Dority JA, Goswami R, Hall PL, Kumar V, Lawrence KB, Logan ME et al. 1992 Synthesis and substance $P$ receptor binding activity of androstano[3,2b]pyrimido[1,2-a]benzimidazoles. Journal of Medicinal Chemistry $\mathbf{3 5}$ 374-378. (doi:10.1021/jm00080a025)

Yu J, Cadet JL \& Angulo JA 2002 Neurokinin-1 (NK-1) receptor antagonists abrogate methamphetamine-induced striatal dopaminergic neurotoxicity in the murine brain. Journal of Neurochemistry 83 613-622. (doi:10.1046/j.1471-4159.2002.01155.x)

Yu YJ, Arttamangkul S, Evans CJ, Williams JT \& von Zastrow M 2009 Neurokinin 1 receptors regulate morphine-induced endocytosis and desensitization of $\mu$-opioid receptors in CNS neurons. Journal of Neuroscience 29 222-233. (doi:10.1523/JNEUROSCI.4315-08.2009)

Zhou W, Li S, Liu Y, Qi X, Chen H, Cheng CH, Liu X, Zhang Y \& Lin H 2012 The evolution of tachykinin/tachykinin receptor (TAC/TACR) in vertebrates and molecular identification of the TAC3/TACR3 system in zebrafish (Danio rerio). Molecular and Cellular Endocrinology 361 202-212. (doi:10.1016/j.mce.2012.04.007)

Received in final form 19 November 2012

Accepted 18 December 2012

Accepted Preprint published online 18 December 2012 http://jme.endocrinology-journals.org DOI: 10.1530/JME-12-0199
() 2013 Society for Endocrinology Printed in Great Britain 